Validity of Edgeworth expansions for realized volatility estimators

\author{
Ulrich Hounyo and Bezirgen Veliyev
}

CREATES Research Paper 2015-21 


\title{
Validity of Edgeworth expansions for realized volatility estimators*
}

\author{
Ulrich Hounyo $^{\dagger}$ \\ Aarhus University, CREATES and Oxford-Man Institute \\ Bezirgen Veliyev $\ddagger$ \\ Aarhus University and CREATES
}

May 3, 2015

\begin{abstract}
The main contribution of this paper is to establish the formal validity of Edgeworth expansions for realized volatility estimators. First, in the context of no microstructure effects, our results rigorously justify the Edgeworth expansions for realized volatility derived in Gonçalves and Meddahi (2009). Second, we show that the validity of the Edgeworth expansions for realized volatility may not cover the optimal two-point distribution wild bootstrap proposed by Gonçalves and Meddahi (2009). Then, we propose a new optimal nonlattice distribution which ensures the second-order correctness of the bootstrap. Third, in the presence of microstructure noise, based on our Edgeworth expansions, we show that the new optimal choice proposed in the absence of noise is still valid in noisy data for the pre-averaged realized volatility estimator proposed by Podolskij and Vetter (2009). Finally, we show how confidence intervals for integrated volatility can be constructed using these Edgeworth expansions for noisy data. Our Monte Carlo simulations show that the intervals based on the Edgeworth corrections have improved the finite sample properties relatively to the conventional intervals based on the normal approximation.
\end{abstract}

Keywords: Realized volatility, pre-averaging, bootstrap, Edgeworth expansions, confidence intervals.

JEL Classification: C15, C22, C58

\section{Introduction}

The increasing availability of complete transaction and quote records for financial assets has spurred a literature seeking to exploit this information in estimating the current level of return volatility. An early popular estimator of integrated volatility is to compute the sum of squared increments of the log price process, i.e. the realized volatility. ${ }^{1}$ An important characteristic of high-frequency financial data is the presence of market microstructure effects: prices are observed with contamination errors (the so-called noise) due to the presence of bid-ask bounce effects, rounding errors, etc., which contribute

${ }^{*}$ We would like to thank Anders Bredahl Kock, Sílvia Gonçalves and Mark Podolskij for many useful comments and discussions on the first version of the paper. We acknowledge support from CREATES - Center for Research in Econometric Analysis of Time Series (DNRF78), funded by the Danish National Research Foundation.

${ }^{\dagger}$ Department of Economics and Business, Aarhus University, Denmark. Email: uhounyo@econ.au.dk.

${ }^{\ddagger}$ Department of Economics and Business, Aarhus University, Denmark. Email: bveliyev@econ.au.dk.

${ }^{1}$ See e.g. the early work by Andersen et al. (2001), Barndorff-Nielsen and Shephard (2002), Comte and Renault (1998), Jacod and Protter (1998), Meddahi (2002), Barndorff-Nielsen et al. (2006) and see Andersen et al. (2010) and Barndorff-Nielsen and Shephard (2007) for reviews. 
to a discrepancy between the latent efficient price process and the price observed by the econometrician. This issue has received a fair amount of attention in the recent literature. Indeed, realized volatility is not consistent for integrated volatility under the presence of market microstructure noise. This has motivated the development of alternative estimators. Currently, there are four main approaches to quadratic variation estimation, namely linear combination of realized volatilities obtained by subsampling (Zhang et al. (2005), and Zhang (2006)), kernel-based autocovariance adjustments (Barndorff-Nielsen et al. (2008)), the pre-averaging method (Podolskij and Vetter (2009), and Jacod et al. (2009)), and the maximum likelihood-based approach (Xiu (2010)).

Recently, Gonçalves and Meddahi (2009) (henceforth GM (2009)) have shown that under general conditions on the price and volatility processes (but excluding microstructure noise), using the bootstrap for inference on volatility could help having better performance than standard asymptotic inference. In particular, GM (2009) have proposed a theoretical justification for using bootstrap for realized volatility. Their simulations confirm the better behavior of the bootstrap method than the asymptotic based approach. Based on Edgeworth expansions, they also provide higher-order refinements of the bootstrap that explain these findings under a stricter set of assumptions that rules out drift, leverage effects and market microstructure effects. However, they do not prove the theoretical validity of their Edgeworth expansions (see GM (2009), footnote 3 on p. 289).

In this paper, we establish the theoretical validity of their Edgeworth expansions. In addition, we show that the validity of the Edgeworth expansions for realized volatility may not cover the optimal two-point distribution wild bootstrap proposed by Gonçalves and Meddahi (2009). Then, we suggest a new optimal external random variable with a density which yields the second-order accuracy of the bootstrap.

Gonçalves et al. (2014) have shown that the wild bootstrap procedure applied on the nonoverlapping pre-averaged returns (as originally proposed by Podolskij and Vetter (2009)) estimates the asymptotic variance as well as the asymptotic mixed normal distribution of the pre-averaged realized volatility estimator. However, for this relatively simple statistic we can simply use, for instance, the consistent variance estimator proposed by Podolskij and Vetter (2009). Hence, the additional effort required for the bootstrap is justified if the resulting approximation to the distribution of the statistic is better than the one relying on the asymptotic normality. With no noisy data, the wild bootstrap studied by GM (2009) indeed has this property. In this paper, we show that this is also true for the wild bootstrap method applied to the non-overlapping pre-averaged returns. Specifically, in the presence of microstructure noise, based on our Edgeworth expansions, we show that the new optimal external random variable with a nonlattice distribution proposed in the absence of noise is still valid in noisy data for the pre-averaging estimator of Podolskij and Vetter (2009).

The main reason for the second-order correctness of the bootstrap procedure in Gonçalves et al. (2014) is the asymptotically correct skewness of the bootstrap distribution. Indeed, an important characteristic of the pre-averaged realized volatility estimator of Podolskij and Vetter (2009) (see also Jacod et al. (2009)) is that it entails an analytical bias correction term. Jacod et al. (2009) have shown that this bias correction is only important for the proper centering of the confidence intervals and does not impact the variance of the estimator. This has motivated Gonçalves et al. (2014) to resample the pre-averaged returns and then to construct bootstrap $t$-statistic without any bias correction term (see also Hounyo et al. (2013) and Hounyo (2013) for closely related proposals for bootstrapping noisy high-frequency financial data). In this paper, we formally show that up to $o\left(n^{-1 / 4}\right)$ (where $n$ is the sample size), the bias correction term does not impact the first three cumulants of the studentized statistic, in particular the skewness of the estimator. As a consequence, the bootstrap method in Gonçalves et al. (2014) does not suffer from the absence of a bias correction term in the bootstrap $t$-statistic at least to consistently estimate the skewness, and more generally in its ability to match the first and third cumulants of pre-averaged realized volatility up to $o\left(n^{-1 / 4}\right)$ (small enough error to yield a second-order refinement). 
Building on Edgeworth expansions for studentized statistics based on the pre-averaged realized volatility estimator, we also propose confidence intervals for integrated volatility that incorporate an analytical correction for skewness as an alternative method of inference. Our approach extends the results in Gonçalves and Meddahi (2008) (henceforth GM (2008)) by allowing for microstructure noise. As in GM (2008), we also find that in a framework where there exist market microstructure effects and the computational burden imposed by the bootstrap is high, using Edgeworth expansions is superior to using the normal approximation derived by Podolskij and Vetter (2009). Our Monte Carlo simulations show that the bootstrap outperforms the Edgeworth corrected intervals. Recently, Zhang et al. (2011) also allow microstructure effects and provided Edgeworth corrections of the normalized statistic (where the denominator equals variance of the estimator in population) rather than studentized statistic (where the denominator is a consistent estimator of the estimator's variance) for several realized measures, including the realized volatility and the noise robust two time scale realized volatility estimator as a mean to improve upon the first order asymptotic. The main reason why we only focus on studentized statistics is because in practice the variance of realized volatility estimators is usually unknown, then studentized statistics are more used. In addition, in the simple framework without market microstructure noise, GM (2008) proved that Edgeworth corrections based on normalized statistic are worse than the asymptotic theory. Edgeworth expansions for realized volatility are also developed by Lieberman and Phillips (2006) for inference on long memory parameters.

A nice side result, which may be useful in other contexts, is that we derive the second-order Edgeworth expansion of a certain form of studentized statistic, where observations are independent but not identically distributed. In particular, observations have a specific heterogeneity properties, which to the best of our knowledge are not covered by other works in the literature. This can be found in Proposition 6.1 in the Appendix.

The remainder of the paper unfolds as follows. The next section briefly introduces the theoretical framework, and the main assumptions. We also review the existing asymptotic theory of realized volatility, in particular, the pre-averaged realized volatility estimator of Podolskij and Vetter (2009). In Section 3, we establish the formal validity of Edgeworth expansions for realized volatility estimators. Section 4 contains Monte Carlo results while Section 5 concludes. All proofs are relegated to the Appendix.

\section{Framework and review of the literature}

We focus on a single asset traded in a liquid financial market. Let $X$ denote the latent efficient logprice process defined on a probability space $(\Omega, \mathcal{F}, P)$ equipped with a filtration $\left(\mathcal{F}_{t}\right)_{t \geq 0}$. We assume that the sample-path of $X$ is continuous and determined by the stochastic differential equation

$$
d X_{t}=b d t+\sigma_{t} d W_{t}, t \geq 0
$$

where $\sigma=\left(\sigma_{t}\right)_{t \geq 0}$ is an adapted càdlàg volatility process, $b$ is a constant drift term and $W=\left(W_{t}\right)_{t \geq 0}$ is a standard Brownian motion. By assumption $W_{t}$ and $\sigma_{t}$ are independent, thus excluding the leverage effect.

The object of interest is the integrated volatility of $X$, i.e. the process

$$
\Gamma_{t}=\int_{0}^{t} \sigma_{s}^{2} d s
$$

Without loss of generality, we let $t=1$ and define $\Gamma=\Gamma_{1}=\int_{0}^{1} \sigma_{s}^{2} d s$ as the integrated volatility of $X$ over the period $[0,1]$, which is thought as a given day.

The availability of market frictions such as bid-ask spreads, price discreteness, rounding errors, etc, hamper us from observing the efficient price process $X$. Instead, we observe a noisy price process 
$Y$, observed at time points $t=\frac{i}{n}$ for $i=0, \ldots, n$, via

$$
Y_{t}=X_{t}+\epsilon_{t},
$$

from which we compute $n$ intraday returns given by

$$
\Delta_{i}^{n} Y \equiv Y_{\frac{i}{n}}-Y_{\frac{i-1}{n}}, i=1, \ldots, n .
$$

where $\epsilon_{t}$ represents the noise term that collects all the market microstructure effects. We impose:

Assumption 1. We suppose that

(i) $\epsilon_{t}$ is i.i.d. with mean 0 and variance $\omega^{2}$. Also, $\mathbb{E}\left[\left|\epsilon_{t}\right|^{2(6+\delta)}\right]<\infty$ for some $\delta>0$.

(ii) $\epsilon_{t}$ is independent of the latent log-price $X_{t}$.

This assumption is standard in the literature related to the noise robust estimators of integrated volatility (see, among others, Zhang et al. (2005), and Barndorff-Nielsen et al. (2008)). However, empirically a decomposition into independent components as in (2) and i.i.d. assumption on noise do not always describe the dynamics of the observed price processes. These assumptions may be too strong especially at the highest frequencies. See e.g. Hansen and Lunde (2006) and Aït-Sahalia et al. (2011) for more on this issue. Most of what we do here could be extended to allow for dependent noise following the details discussed in Gonçalves et al. (2014). But, an exploration of this extended setting is left for future research.

Next, we introduce an additional regularity condition on the volatility path. In particular, we follow Barndorff-Nielsen and Shephard (2003), see also GM (2009), and make the following assumption.

Assumption 2. The volatility $\sigma$ is a càdlàg process, bounded away from zero, and satisfies

$$
\lim _{n \rightarrow \infty} n^{-1 / 2} \sum_{i=1}^{n}\left|\sigma_{\eta_{i}}^{r}-\sigma_{\xi_{i}}^{r}\right|=0
$$

for some $r>0$ and for any $\eta_{i}$ and $\xi_{i}$ with $0 \leq \xi_{1} \leq \eta_{1} \leq n^{-1} \leq \xi_{2} \leq \eta_{2} \leq 2 n^{-1} \leq \ldots \leq \xi_{n} \leq \eta_{n} \leq 1$.

Assumption 2 is stronger than required to prove the central limit theorem for the integrated volatility estimator, but it is a convenient assumption to derive Edgeworth expansions. Relaxing this assumption is beyond the scope of this paper. In view of footnote 2 in Barndorff-Nielsen and Shephard (2003), we note that if Assumption 2 holds for some $r>0$, then it holds for any $r>0$.

In the following, we denote by $\widehat{\Gamma}_{n}$ a consistent estimator of the integrated volatility $\Gamma$ such that a central limit theorem holds with the convergence rate of $\tau_{n}$. In particular, we have as $n \rightarrow \infty$

$$
T_{n} \equiv \frac{\tau_{n}\left(\widehat{\Gamma}_{n}-\Gamma\right)}{\sqrt{\hat{V}_{n}}} \stackrel{d}{\rightarrow} N(0,1),
$$

where $\hat{V}_{n}$ is a consistent estimator of the asymptotic variance $V$ of $\tau_{n} \widehat{\Gamma}_{n}$. As statistics of interest in this paper, we focus on the realized volatility and the pre-averaging estimator of Podolskij and Vetter (2009).

We first review the existing results. While in the paper we also analyzed finite sample behavior of the pre-averaging estimator based on data at the highest frequency, the setting of moderate frequencies serves as an important benchmark. We start with this benchmark case due to its relative simplicity. 


\subsection{Realized volatility estimator}

In this subsection, we consider the simple case where no market microstructure noise exists $(\epsilon \equiv 0)$. It follows that $Y=X$, where $X$ follows (1). In applied work, this refers to a situation where the sampling frequencies are low enough for the effects of market microstructure to be negligible, e.g. 5, 10, or 30 minutes. In this relatively simple scenario, a popular consistent estimator of integrated volatility is the realized volatility (see e.g. Barndorff-Nielsen and Shephard (2002)). Barndorff-Nielsen et al. (2006) derived a feasible central limit theorem for realized volatility defined by

$$
\widehat{\Gamma}_{n}=\sum_{i=1}^{n}\left(\Delta_{i}^{n} Y\right)^{2} .
$$

They showed that, as $n \rightarrow \infty$, (4) holds, under very general conditions which allow the presence of time varying drift as well as leverage effects, for the statistic $T_{n}$ defined as

$$
T_{n}=\frac{\sqrt{n}\left(\sum_{i=1}^{n}\left(\Delta_{i}^{n} Y\right)^{2}-\Gamma\right)}{\sqrt{\frac{2}{3} n \sum_{i=1}^{n}\left(\Delta_{i}^{n} Y\right)^{4}}} .
$$

We can use this feasible asymptotic distribution result to build confidence intervals for integrated volatility. In particular, the conventional $100(1-\alpha) \%$ level one-sided confidence interval for $\Gamma$ is given by:

$$
I C_{\text {Feas }, 1-\alpha}^{A T-1}=\left(-\infty, \widehat{\Gamma}_{n}-\tau_{n}^{-1} \sqrt{\hat{V}_{n}} z_{\alpha}\right),
$$

whereas a two-sided symmetric feasible $100(1-\alpha) \%$ level interval for $\Gamma$ is given by:

$$
I C_{\text {Feas }, 1-\alpha}^{A T-2}=\left(\widehat{\Gamma}_{n}-z_{1-\alpha / 2} \tau_{n}^{-1} \sqrt{\hat{V}_{n}}, \widehat{\Gamma}_{n}+z_{1-\alpha / 2} \tau_{n}^{-1} \sqrt{\hat{V}_{n}}\right)
$$

where $z_{1-\alpha / 2}$ is such that $\Phi\left(z_{1-\alpha / 2}\right)=1-\alpha / 2$, and $\Phi(\cdot)$ is the cumulative distribution function of the standard normal distribution. For instance, $z_{0.05}=-1.645$ and $z_{0.975}=1.96$ when $\alpha=0.05$. As GM (2009) have shown, in finite sample, this approach can lead to important coverage distorsions. As a remedy, GM (2008) suggested to use Edgeworth corrected confidence intervals for realized volatility. Whereas, GM (2009) proposed confidence intervals based on bootstrap methods for $\widehat{\Gamma}_{n}$. In particular, following GM (2008) we can obtain, for instance, an improved symmetric confidence interval for $\Gamma$ relying on Edgeworth expansion of $T_{n}$. We will study these intervals in detail in Section 3. For the bootstrap, GM's (2009) wild bootstrap method for realized volatility resamples as follows

$$
\Delta_{i}^{n} Y^{*}=\Delta_{i}^{n} Y \cdot v_{i}, \quad i=1, \ldots, n .
$$

where the external random variable $v_{i}$ is an i.i.d. random variable independent of the data and whose moments are given by $a_{q}^{*} \equiv \mathbb{E}^{*}\left[\left|v_{i}\right|^{q}\right]$. As usual in the bootstrap literature, $\mathbb{P}^{*}, \mathbb{E}^{*}$ and $V a r^{*}$ denote the probability measure, expected value and variance induced by the bootstrap resampling, conditional on a realization of the original time series, respectively. In addition, for a sequence of bootstrap statistics $Z_{n}^{*}$, we write $Z_{n}^{*}=o_{p^{*}}(1)$ in probability, or $Z_{n}^{*} \stackrel{\mathbb{P}^{*}}{\rightarrow} 0$, as $n \rightarrow \infty$, in probability, if for any $\varepsilon>0, \delta>0$, $\lim _{n \rightarrow \infty} \mathbb{P}\left[\mathbb{P}^{*}\left[\left|Z_{n}^{*}\right|>\delta\right]>\varepsilon\right]=0$. Similarly, we write $Z_{n}^{*}=O_{p^{*}}(1)$ as $n \rightarrow \infty$, in probability if for all $\varepsilon>0$ there exists a $M_{\varepsilon}<\infty$ such that $\lim _{n \rightarrow \infty} \mathbb{P}\left[\mathbb{P}^{*}\left[\left|Z_{n}^{*}\right|>M_{\varepsilon}\right]>\varepsilon\right]=0$. Finally, we write $Z_{n}^{*} \stackrel{d^{*}}{\rightarrow} Z$ as $n \rightarrow \infty$, in probability, if conditional on the sample, $Z_{n}^{*}$ converges weakly to $Z$ under $\mathbb{P}^{*}$, for all samples contained in a set with probability $\mathbb{P}$ converging to one. 
Then, based on bootstrap returns $\Delta_{i}^{n} Y^{*}$, GM (2009) defined the bootstrap realized volatility analogue of $\widehat{\Gamma}_{n}$ as $\widehat{\Gamma}_{n}^{*}=\sum_{i=1}^{n}\left(\Delta_{i}^{n} Y^{*}\right)^{2}$. They showed that, as $n \rightarrow \infty$

$$
T_{n}^{*} \equiv \frac{\sqrt{n}\left(\widehat{\Gamma}_{n}^{*}-a_{2}^{*} \widehat{\Gamma}_{n}\right)}{\sqrt{\hat{V}_{n}^{*}}} \stackrel{d^{*}}{\rightarrow} N(0,1)
$$

where $\hat{V}_{n}^{*}=\frac{\left(a_{4}^{*}-a_{2}^{* 2}\right)}{a_{4}^{*}} n \sum_{i=1}^{n}\left(\Delta_{i}^{n} Y^{*}\right)^{4}$. This result justifies constructing bootstrap percentile- $t$ (bootstrap studentized statistic) intervals. In particular, a $100(1-\alpha) \%$ one sided bootstrap percentile- $t$ interval for integrated volatility is given by

$$
I C_{\text {perc- } t, 1-\alpha}^{* B-1}=\left(-\infty, \widehat{\Gamma}_{n}-\tau_{n}^{-1} \sqrt{\hat{V}_{n}} z_{\alpha}^{* B-1}\right)
$$

whereas a $100(1-\alpha) \%$ symmetric bootstrap percentile- $t$ interval for integrated volatility is given by

$$
I C_{\text {perc }-t, 1-\alpha}^{* B-2}=\left(\widehat{\Gamma}_{n}-z_{1-\alpha}^{* B-2} \tau_{n}^{-1} \sqrt{\hat{V}_{n}}, \widehat{\Gamma}_{n}+z_{1-\alpha}^{* B-2} \tau_{n}^{-1} \sqrt{\hat{V}_{n}}\right)
$$

where $z_{\alpha}^{* B-1}$ is the $\alpha$-quantile of the bootstrap distribution of $T_{n}^{*}$ whereas $z_{1-\alpha}^{* B-2}$ is the $(1-\alpha)$-quantile of the bootstrap distribution of $\left|T_{n}^{*}\right|$. Next we review the existing results of Podolskij and Vetter's (2009) pre-averaged realized volatility estimator.

\subsection{The pre-averaged estimator and its asymptotic theory}

We now turn to the case where market microstructure effects are not negligible $(\epsilon \neq 0)$. Given that $Y=X+\epsilon$, we can write

$$
\Delta_{i}^{n} Y=\left(X_{\frac{i}{n}}-X_{\frac{i-1}{n}}\right)+\left(\epsilon_{\frac{i}{n}}-\epsilon_{\frac{i-1}{n}}\right) \equiv \Delta_{i}^{n} X+\Delta_{i}^{n} \epsilon,
$$

where $\Delta_{i}^{n} X$ denotes the $\frac{1}{n}$-frequency return on the efficient price process. Under Assumption 1 , the order of magnitude of $\Delta_{i}^{n} \epsilon$ is $O_{p}(1)$. In contrast, $\Delta_{i}^{n} X$ is asymptotically uncorrelated and heteroskedastic with (conditional) variance given by $\int_{(i-1) / n}^{i / n} \sigma_{s}^{2} d s$. Thus, its order of magnitude is $O_{p}\left(n^{-1 / 2}\right)$. This decomposition shows that the noise completely dominates the observed return process as $n \rightarrow \infty$, implying that the usual realized volatility estimator is biased and inconsistent. See, e.g., Zhang et al. (2005) and Bandi and Russell (2008).

As mentioned in the introductory section, there are several estimators of realized volatility that explicitly take microstructure noise effects into account. We consider the non-overlapping pre-averaging estimator of Podolskij and Vetter (2009). To describe this approach, let $k_{n}$ be a sequence of integers which will denote the window length over which the pre-averaging of returns is done. Similarly, let $g$ be a weighting function on $[0,1]$ such that $g(0)=g(1)=0$ and $\int_{0}^{1} g(s)^{2} d s>0$, and assume $g$ is continuous and piecewise continuously differentiable with a piecewise Lipschitz derivative $g^{\prime}$. An example of a function that satisfies these restrictions is $g(x)=\min (x, 1-x)$. We also introduce

$$
\psi_{1}^{k_{n}}=k_{n} \sum_{i=1}^{k_{n}}\left(g\left(\frac{i}{k_{n}}\right)-g\left(\frac{i-1}{k_{n}}\right)\right)^{2} \text { and } \psi_{2}^{k_{n}}=\frac{1}{k_{n}} \sum_{i=1}^{k_{n}} g^{2}\left(\frac{i}{k_{n}}\right) .
$$

These quantities have the following limits

$$
\psi_{1}^{k_{n}}=\psi_{1}+o\left(n^{-1 / 4}\right) \text { and } \psi_{2}^{k_{n}}=\psi_{2}+o\left(n^{-1 / 4}\right),
$$


where

$$
\psi_{1}=\int_{0}^{1}\left(g^{\prime}(s)\right)^{2} d s \text { and } \psi_{2}=\int_{0}^{1}(g(s))^{2} d s .
$$

For instance, for $g(x)=\min (x, 1-x)$, we have that $\psi_{1}=1$ and $\psi_{2}=1 / 12$.

For $i=0, \ldots, n-k_{n}+1$, the pre-averaged returns $\bar{Y}_{i}$ are obtained by computing the weighted sum of all consecutive $\frac{1}{n}$-horizon returns over each block of size $k_{n}$,

$$
\bar{Y}_{i}=\sum_{j=1}^{k_{n}} g\left(\frac{j}{k_{n}}\right) \Delta_{i+j}^{n} Y .
$$

The aim of pre-averaging is to control the stochastic orders of the pre-averaged terms via $k_{n}$. In particular, we get

$$
\bar{X}_{i}=\sum_{j=1}^{k_{n}} g\left(\frac{j}{k_{n}}\right)\left(X_{\frac{i+j}{n}}-X_{\frac{i+j-1}{n}}\right)=O_{p}\left(\frac{\sqrt{k_{n}}}{\sqrt{n}}\right),
$$

and

$$
\bar{\epsilon}_{i}=\sum_{j=1}^{k_{n}} g\left(\frac{j}{k_{n}}\right)\left(\epsilon_{\frac{i+j}{n}}-\epsilon_{\frac{i+j-1}{n}}\right)=O_{p}\left(\frac{1}{\sqrt{k_{n}}}\right) .
$$

Thus, the impact of the noise is reduced the larger $k_{n}$ is. We put the following condition on $k_{n}$ :

Assumption 3. We suppose that

(i) There exists $\theta \in(0, \infty)$ such that

$$
\frac{k_{n}}{\sqrt{n}}=\theta+o\left(n^{-1 / 4}\right)
$$

(ii) For any $n \geq 1, k_{n}$ divides $n$.

Assumption 3(i) is standard in the literature (see Jacod et al. (2009)). This choice implies that the orders of the terms $\bar{X}_{i}$ and $\bar{\epsilon}_{i}$ are balanced and equal to $O_{p}\left(n^{-1 / 4}\right)$. An example that satisfies (15) is $k_{n}=[\theta \sqrt{n}]$. Assumption 3(ii) is imposed in this work to deal with the Edgeworth expansion.

Podolskij and Vetter (2009) propose the following estimator of integrated volatility:

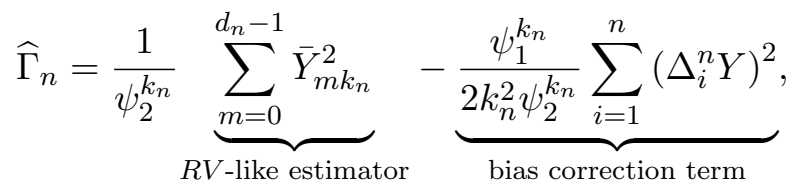

where $d_{n} \equiv n / k_{n}$ and $\psi_{1}^{k_{n}}, \psi_{2}^{k_{n}}$ are as in (13).

The pre-averaging estimator is then simply the analogue of the realized volatility but based on pre-averaged returns and an additional term to remove the bias due to noise. As discussed in Jacod et al. (2009) and Gonçalves et al. (2014), this bias term does not contribute to the asymptotic variance of $\widehat{\Gamma}_{n}$. One of our contributions is to show that at second-order this bias term does not impact the asymptotic distribution of $\widehat{\Gamma}_{n}$ but possibly at third-order its impact may be important.

Under Assumptions 1 and 3, Podolskij and Vetter (2009) show that $\widehat{\Gamma}_{n}$ given by (16) satisfies a central limit theorem as in (4) with $\tau_{n}=n^{1 / 4}$. In particular, the $t$-staistic is

$$
T_{n}=\frac{n^{1 / 4}\left(\widehat{\Gamma}_{n}-\Gamma\right)}{\sqrt{\hat{V}_{n}}}
$$


where the asymptotic conditional variance $V$ and $\hat{V}_{n}$ (an estimator of $V$ ) are respectively given by

$$
V=\frac{2}{\theta \psi_{2}^{2}} \int_{0}^{1}\left(\theta \psi_{2} \sigma_{s}^{2}+\frac{\psi_{1}}{\theta} \omega^{2}\right)^{2} d s, \text { and } \hat{V}_{n}=\frac{2 \sqrt{n}}{3\left(\psi_{2}^{k_{n}}\right)^{2}} \sum_{m=0}^{d_{n}-1} \bar{Y}_{m k_{n}}^{4}
$$

Recently, Gonçalves et al. (2014) have shown that a wild bootstrap procedure applied to the nonoverlapping pre-averaged returns $\bar{Y}_{m k_{n}}$ estimates the asymptotic variance $V$ as well as the asymptotic mixed normal distribution of the pre-averaged realized volatility estimator $\widehat{\Gamma}_{n}$. More specifically, Gonçalves et al. (2014) suggested to resample as follows

$$
\bar{Y}_{m k_{n}}^{*}=\bar{Y}_{m k_{n}} \cdot v_{m}, \quad m=0, \ldots, d_{n}-1 .
$$

where the external random variable $v_{m}$ is an i.i.d. random variable independent of the data and whose moments are given by $a_{q}^{*}=\mathbb{E}^{*}\left[\left|v_{m}\right|^{q}\right]$. Then based on bootstrap pre-averaged returns $\bar{Y}_{m k_{n}}^{*}$, Gonçalves et al. (2014) defined the bootstrap pre-averaged realized volatility estimator as $\widehat{\Gamma}^{n *}=\frac{1}{\psi_{2}^{k_{n}}} \sum_{m=0}^{d_{n}-1} \bar{Y}_{m k_{n}}^{* 2}$. They show that, as $n \rightarrow \infty$

$$
T_{n}^{*} \equiv \frac{n^{1 / 4}\left(\widehat{\Gamma}_{n}^{*}-\mathbb{E}^{*}\left(\widehat{\Gamma}_{n}^{*}\right)\right)}{\sqrt{\hat{V}_{n}^{*}}} \stackrel{d^{*}}{\rightarrow} N(0,1)
$$

where $\mathbb{E}^{*}\left(\widehat{\Gamma}_{n}^{*}\right)=\frac{a_{2}^{*}}{\psi_{2}^{k_{n}}} \sum_{m=0}^{d_{n}-1} \bar{Y}_{m k_{n}}^{2}$ and $\hat{V}_{n}^{*}=\frac{\left(a_{4}^{*}-a_{2}^{* 2}\right)}{a_{4}^{*}\left(\psi_{2}^{k_{n}}\right)^{2}} \sqrt{n} \sum_{m=0}^{d_{n}-1} \bar{Y}_{m k_{n}}^{* 4}$. This justifies constructing bootstrap percentile- $t$ intervals for integrated volatility in the presence of noise.

Note that although in (16) $\widehat{\Gamma}_{n}$ contains a bias correction term, it is not the case for $\widehat{\Gamma}^{n *}$. As they argue, this is because the bias correction term by definition does not affect at first order the asymptotic variance of $\widehat{\Gamma}_{n}$. In the next section we will investigate the impact of the bias correction term on the first three cumulants of studentized statistic up to $o\left(n^{-1 / 4}\right)$.

\section{Edgeworth expansion for realized volatility}

Here we establish the validity of formal Edgeworth expansions for $\widehat{\Gamma}_{n}$, where $\widehat{\Gamma}_{n}$ is given either by (5) or (16). Our results apply to the $t$-statistic $T_{n}$ and the bootstrap $t$-statistic $T_{n}^{*}$ defined above. We start by studying the no noise case.

\subsection{Edgeworth expansion without noise}

To describe the Edgeworth expansion, we need to introduce additional notation. To facilitate comparison, we keep the notation of GM (2009) whenever possible. For any $r, s>0$, we let $R_{r, s}=R_{r} / R_{s}^{r / s}$ and $\sigma_{r, s}=\bar{\sigma}^{r} /\left(\bar{\sigma}^{s}\right)^{r / s}$ where

$$
R_{r}=n^{\frac{r}{2}-1} \sum_{i=1}^{n}\left|\Delta_{i}^{n} Y\right|^{r} \quad \text { and } \bar{\sigma}^{r}=\int_{0}^{1} \sigma_{t}^{r} d t
$$

Similarly, we let $a_{r, s}=a_{r} / a_{s}^{r / s}$ where $a_{s}=\mathbb{E}\left[|U|^{s}\right]$ such that $U \sim N(0,1)$.

Theorem 3.1. Suppose (1) holds with $b=0$. Under Assumption 2, conditionally on $\sigma$, the secondorder Edgeworth expansions of the studentized statistics $T_{n}$ and $T_{n}^{*}$ defined in (6) and (10), respectively, are given by 
(1)

$$
\mathbb{P}\left[T_{n} \leq x\right]=\Phi(x)+n^{-1 / 2} q_{1}(x) \phi(x)+o\left(n^{-1 / 2}\right)
$$

where

$$
q_{1}(x)=\left(\frac{A_{1}}{2}-\frac{1}{6}\left(B_{1}-3 A_{1}\right)\left(x^{2}-1\right)\right) \sigma_{6,4},
$$

with

$$
A_{1}=\frac{a_{6}-a_{2} a_{4}}{a_{4}\left(a_{4}-a_{2}^{2}\right)^{1 / 2}}=\frac{4}{\sqrt{2}} \text { and } B_{1}=\frac{a_{6}-3 a_{2} a_{4}+2 a_{2}^{3}}{\left(a_{4}-a_{2}^{2}\right)^{3 / 2}}=\frac{4}{\sqrt{2}} .
$$

(2) In addition, suppose that $\left\{\Delta_{j}^{n} Y^{*}=\Delta_{j}^{n} Y \cdot v_{j}, j=1, \ldots, n\right\}$, where $v_{j} \sim$ i.i.d. whose moments are given by $a_{s}^{*}=\mathbb{E}^{*}\left|v_{j}\right|^{s}$ with $a_{2(6+\delta)}^{*}<\infty$ for some $\delta>0$ and $v_{j}$ 's satisfy Cramer's condition. That is, for all $r>0$, there exists $M_{r} \in(0,1)$ such that

$$
\left|\phi_{n, j}(t)\right| \leq M_{r} \text { for all }\|t\| \geq r \text { and } n \geq 1,1 \leq j \leq n,
$$

where $\phi_{n, j}$ is the characteristic function of $\left(n\left|\Delta_{j}^{n} Y\right|^{2} v_{j}^{2}, n^{2}\left|\Delta_{j}^{n} Y\right|^{4} v_{j}^{4}\right)^{\prime}$ under $\mathbb{P}^{*}$. Then

$$
\mathbb{P}^{*}\left[T_{n}^{*} \leq x\right]=\Phi(x)+n^{-1 / 2} q_{1}^{*}(x) \phi(x)+o_{p}\left(n^{-1 / 2}\right)
$$

where

$$
q_{1}^{*}(x)=\left(\frac{A_{1}^{*}}{2}-\frac{1}{6}\left(B_{1}^{*}-3 A_{1}^{*}\right)\left(x^{2}-1\right)\right) R_{6,4},
$$

with

$$
A_{1}^{*}=\frac{a_{6}^{*}-a_{2}^{*} a_{4}^{*}}{a_{4}^{*}\left(a_{4}^{*}-a_{2}^{* 2}\right)^{1 / 2}} \text { and } B_{1}^{*}=\frac{a_{6}^{*}-3 a_{2}^{*} a_{4}^{*}+2 a_{2}^{* 3}}{\left(a_{4}^{*}-a_{2}^{* 2}\right)^{3 / 2}}
$$

We note that these results are derived under the same assumptions as in Proposition 4.1 of GM (2009). Since we have shown the validity of our Edgeworth expansions in this paper, our results justify GM's (2009) Proposition 4.1. In contrast to GM (2009) (cf. footnote 3 on p. 289), we do not assume the existence of Edgeworth expansions derived in (21) and (24), rather we formally verify conditions under which these Edgeworth expansions exist (since some cumulants may be infinite).

Remark 1. Unfortunately, the best existing choice of $v_{j}$ (i.e, the optimal two-point distribution) suggested in Proposition 4.5 of GM (2009) does not satisfy condition (23) in part (2) of Theorem 3.1 and hence it is unlikely that the second-order Edgeworth expansions of the bootstrap studentized statistic $T_{n}^{*}$ exists for this choice.

In view of above remark, we suggest a distribution that has a density.

Proposition 3.1. Let $T_{n}$ and $T_{n}^{*}$ be defined as in (6) and (10), respectively. Morover, $v_{1}, \ldots, v_{n}$ as defined in (9) be i.i.d. with $v_{i}=\sqrt{\eta_{i}}$ where $\eta_{i}$ has the gamma density

$$
f(x)=\frac{\beta^{\alpha}}{\Gamma(\alpha)} x^{\alpha-1} \exp (-\beta x) I_{(x>0)}
$$

with $\alpha=\beta=\frac{25}{6}$. Suppose (1) holds with $b=0$. Under Assumption 2, conditionally on $\sigma$, as $n \rightarrow \infty$

$$
\sup _{x \in \mathbb{R}}\left|\mathbb{P}^{*}\left[T_{n}^{*} \leq x\right]-\mathbb{P}\left[T_{n} \leq x\right]\right|=o_{p}\left(n^{-1 / 2}\right) .
$$


Remark 2. The square root term in the optimal choice of the external random variable in Proposition 3.1 suggests the following modification of the wild bootstrap procedure proposed by GM (2009). We propose to resample directly the square returns $\left(\Delta_{i}^{n} Y\right)^{2}$ instead of the raw returns $\Delta_{i}^{n} Y$ :

$$
\left(\Delta_{i}^{n} Y^{*}\right)^{2}=\left(\Delta_{i}^{n} Y\right)^{2} \cdot\left|\eta_{i}\right|, i=1, \ldots, n,
$$

where as before the external random variable $\eta_{i}$ is an i.i.d. random variable independent of the data and whose moments are given by $a_{q}^{*}=\mathbb{E}^{*}\left[\left|\eta_{i}\right|^{q}\right]$. For the second-order accuracy of the bootstrap, GM(2009) imposed conditions on the first even moments ( $a_{2}^{*}, a_{4}^{*}$ and $a_{6}^{*}$ ) of the external random variable $v$, whereas with the new wild bootstrap we require conditions on the first three moments $\left(a_{1}^{*}, a_{2}^{*}\right.$ and $\left.a_{3}^{*}\right)$ of $\eta_{i}$. Then, the gamma distribution choice of $\eta_{i}$ defined in Proposition 3.1 provides a second-order asymptotic refinement.

So far we have focused on the case $b=0$. In the following remark, we allow a non-zero drift term.

Remark 3. Suppose (1) holds with $b \neq 0$. Under Assumption 2, conditionally on $\sigma$, the second-order formal Edgeworth expansion of the studentized statistic $T_{n}$ defined in (6) (assuming the corresponding Edgeworth expansion exists) is given by

$$
\mathbb{P}\left[T_{n} \leq x\right]=\Phi(x)+n^{-1 / 2} q_{1}(x) \phi(x)+o\left(n^{-1 / 2}\right),
$$

where

$$
q_{1}(x)=\left(\frac{A_{1}}{2}-\frac{1}{6}\left(B_{1}-3 A_{1}\right)\left(x^{2}-1\right)\right) \sigma_{6,4}-\frac{b^{2}}{\bar{\sigma}_{4}},
$$

with $A_{1}$ and $B_{1}$ defined as in Theorem 3.1, in particular $A_{1}=B_{1}=4 / \sqrt{2}$.

Assuming that the corresponding Edgeworth expansions exist, Remark 3 emphasized that the effect of the drift on $T_{n}$ is not negligible at second-order. In particular, a comparison of equations (22) and (27) shows that an additional term $-\frac{b^{2}}{\bar{\sigma}_{4}}$ shows up in (27) when $b \neq 0$. At first-order one can show that the effect of the drift on $T_{n}$ is $O_{p}\left(n^{-1 / 2}\right)$, that is negligible.

Remark 4. As highlighted in GM (2009), results in Theorems 3.1 are not special cases of Liu (1988). She derived the second-order Edgeworth expansions of the studentized statistic defined by

$$
T_{n}=\frac{\sqrt{n}\left(n^{-1} \sum_{i=1}^{n} \mathcal{Z}_{i}-n^{-1} \sum_{i=1}^{n} \mathbb{E}\left[\mathcal{Z}_{i}\right]\right)}{\sqrt{\hat{V}_{n}}},
$$

where $\mathcal{Z}_{1}, \ldots, \mathcal{Z}_{n}$ are a set of independent but not identical random observations with the sample variance $\hat{V}_{n}=n^{-1} \sum_{i=1}^{n} \mathcal{Z}_{i}^{2}-\left(n^{-1} \sum_{i=1}^{n} \mathcal{Z}_{i}\right)^{2}$. She also showed the second-order properties of Wu's (1986) weighted bootstrap, the so called wild bootstrap procedure. The differences between Liu's (1988) work and results in Theorem 3.1 are at least twofold. First, her results apply to $t$ and bootstrap $t$ statistics that are both studentized by the sample variance. In particular, in part (1) of Theorem 3.1, we would be able to use Liu's (1988) results in the context of realized volatility (with no noise), if instead of using the studentized statistics $t$ defined in (6) we have considered the following $t$ statistic:

$$
T_{n}=\frac{\sqrt{n}\left(R_{2}-\Gamma\right)}{\sqrt{R_{4}-R_{2}^{2}}},
$$

where $R_{r}$ (with $r=2,4$ ) is given by (20). It is easy to see that, letting $\mathcal{Z}_{i} \equiv n\left|\Delta_{i}^{n} Y\right|^{2}$, we can write $R_{2}=n^{-1} \sum_{i=1}^{n} \mathcal{Z}_{i}$ and the sample variance estimator of $\sqrt{n} R_{2}, \hat{V}_{n}=R_{4}-R_{2}^{2}=n^{-1} \sum_{i=1}^{n} \mathcal{Z}_{i}^{2}-$ $\left(n^{-1} \sum_{i=1}^{n} \mathcal{Z}_{i}\right)^{2}$. Unfortunately, we cannot use $R_{4}-R_{2}^{2}$ to studentize realized volatility when volatility 
is time-varying. Second, Liu's (1988) wild bootstrap is applied on centered observations. In particular, in order to use Liu's (1988) second-order Edgeworth expansions for the bootstrap $t$ statistic, the wild bootstrap observations should be resampled as follows

$$
\mathcal{Z}_{i}^{*}=n^{-1} \sum_{i=1}^{n} \mathcal{Z}_{i}-\left(\mathcal{Z}_{i}-n^{-1} \sum_{i=1}^{n} \mathcal{Z}_{i}\right) v_{i}, i=1, \ldots, n
$$

where $\mathcal{Z}_{i}=n\left|\Delta_{i}^{n} Y\right|^{2}$ and $v_{i} \sim$ i.i.d with mean 0 and variance 1 . We observe that this is different from GM's (2009) wild bootstrap method suggested for realized volatility. The t-statistics defined in (6) and (10) are our statistics of interest here and these are not covered by results in Liu (1988).

\subsection{Edgeworth expansion for the pre-averaging estimator}

First, we introduce notations. For any $r, s>0$, we let $\widetilde{R}_{r, s}=\widetilde{R}_{r} / \widetilde{R}_{s}^{r / s}$ and $\widetilde{\sigma}_{r, s}=\widetilde{\sigma}^{r} /\left(\widetilde{\sigma}^{s}\right)^{r / s}$ where

$$
\widetilde{R}_{r}=\frac{1}{\left(\psi_{2}^{k_{n}}\right)^{r / 2}} n^{\frac{r}{4}-\frac{1}{2}} \sum_{m=0}^{d_{n}-1}\left|\bar{Y}_{m k_{n}}\right|^{r}, \quad \text { and } \widetilde{\sigma}^{r}=\int_{0}^{1}\left(\sigma_{t}^{2}+\frac{\omega^{2} \psi_{1}}{\theta^{2} \psi_{2}}\right)^{r / 2} d t
$$

Furthermore, we denote

$$
s_{i}^{2} \equiv \sum_{j=1}^{k_{n}-1} g^{2}\left(\frac{j}{k_{n}}\right) \int_{\frac{(i-1) k_{n}+j-1}{n}}^{\frac{(i-1) k_{n}+j}{n}} \sigma_{t}^{2} d t
$$

Note that, conditionally on $\sigma, s_{i}^{2}$ is the expectation of $\left(\bar{X}_{(i-1) k_{n}}\right)^{2}$. We also let

$$
Z_{d_{n}, i}=\frac{d_{n}}{\psi_{2}^{k_{n}}}\left(\bar{Y}_{(i-1) k_{n}}\right)^{2}, \mu_{d_{n}, i}=\frac{d_{n}}{\psi_{2}^{k_{n}}}\left(s_{i}^{2}+\frac{\psi_{1}^{k_{n}} \omega^{2}}{k_{n}}\right), B_{d_{n}, i}=\frac{\psi_{1}^{k_{n}} d_{n}}{2 k_{n}^{2} \psi_{2}^{k_{n}}} \sum_{j=(i-1) k_{n}+1}^{i k_{n}-1}\left(\left|\Delta_{j}^{n} \epsilon\right|^{2}-2 \omega^{2}\right) .
$$

To state our Edgeworth expansion results for pre-averaged realized volatility, we require a slightly stronger condition on the volatility $\sigma$ than Assumption 2. Specifically, we impose:

Assumption 4. The volatility $\sigma$ is a càdlàg process, bounded away from zero, and satisfies the following regularity condition: For some $\delta>0$, we have

$$
\frac{1}{\psi_{2}^{k_{n}}} \sum_{i=1}^{d_{n}} s_{i}^{2}-\int_{0}^{1} \sigma_{t}^{2}=O\left(n^{-1 / 2-\delta}\right) .
$$

To ensure this assumption, one can for example suppose that $\sigma_{t}$ is pathwise Lipschitz continuous. Next, we also require Cramer's condition for $\widetilde{A}_{m, i}$. In particular, we assume:

Assumption 5. For all $r>0$, there exists $M_{r} \in(0,1)$ such that

$$
\left|\phi_{d_{n}, i}(t)\right| \leq M_{r} \text { for all }\|t\| \geq r \text { and } d_{n} \geq 1,1 \leq i \leq d_{n}
$$

where $\phi_{d_{n}, i}$ is the characteristic function of $\left(Z_{d_{n}, i}-\mu_{d_{n}, i}-B_{d_{n}, i}, Z_{d_{n}, i}^{2}-\mathbb{E}\left[Z_{d_{n}, i}^{2}\right]\right)^{\prime}$.

Under above conditions, the following theorem holds true.

Theorem 3.2. Suppose (1) holds with $b=0$. Under Assumptions 1, 3, 4 and 5 and conditionally on $\sigma$, the formal second-order Edgeworth expansions of the studentized statistics $T_{n}$ and $T_{n}^{*}$ defined in (17) and (19), respectively, are given by 
(1)

$$
\mathbb{P}\left[T_{n} \leq x\right]=\Phi(x)+n^{-1 / 4} q_{1}(x) \phi(x)+o\left(n^{-1 / 4}\right)
$$

where

$$
q_{1}(x)=\left(\frac{A_{1}}{2}-\frac{1}{6}\left(B_{1}-3 A_{1}\right)\left(x^{2}-1\right)\right) \widetilde{\sigma}_{6,4}
$$

with $A_{1}$ and $B_{1}$ defined as in Theorem 3.1, in particular $A_{1}=B_{1}=4 / \sqrt{2}$.

(2) In addition, suppose that $\left\{\bar{Y}_{m k_{n}}^{*}=\bar{Y}_{m k_{n}} \cdot v_{m}, m=0, \ldots, d_{n}-1\right\}$, where $v_{m} \sim$ i.i.d whose moments are given by $a_{s}^{*}=\mathbb{E}^{*}\left[\left|v_{m}\right|^{s}\right]$ with $a_{2(6+\delta)}^{*}<\infty$ for some $\delta>0$ and $v_{m}$ 's satisfy Cramer's condition. Namely, for all $r>0$, there exists $M_{r} \in(0,1)$ such that

$$
\left|\phi_{d_{n}, m}(t)\right| \leq M_{r} \text { for all }\|t\| \geq r \text { and } d_{n} \geq 1,0 \leq m \leq d_{n}-1,
$$

where $\phi_{d_{n}, m}$ is the characteristic function of $\left(d_{n}\left|\bar{Y}_{m k_{n}}\right|^{2} v_{m}^{2}, d_{n}^{2}\left|\bar{Y}_{m k_{n}}\right|^{4} v_{m}^{4}\right)^{\prime}$ under $\mathbb{P}^{*}$. Then

$$
\mathbb{P}^{*}\left[T_{n}^{*} \leq x\right]=\Phi(x)+n^{-1 / 4} q_{1}^{*}(x) \phi(x)+o_{p}\left(n^{-1 / 4}\right)
$$

where

$$
q_{1}^{*}(x)=\left(\frac{A_{1}^{*}}{2}-\frac{1}{6}\left(B_{1}^{*}-3 A_{1}^{*}\right)\left(x^{2}-1\right)\right) \widetilde{R}_{6,4}
$$

with $A_{1}^{*}$ and $B_{1}^{*}$ defined as in Theorem 3.1.

Theorem 3.2 extends Proposition 4.1 of GM (2009) to the noisy setting by utilizing the pre-averaged realized volatility estimator of Podolskij and Vetter (2009). In contrast to the no noise case, we require the Cramer's condition for the validity of Theorem 3.2 in addition to the regularity conditions on $\sigma$. The verification of this Cramer's condition under even the i.i.d. noise assumption as in Assumption 1 may involve nontrivial technical work. The added challenge is readily illustrated by computing the distribution of $Z_{m, i}-B_{m, i}$ in a toy model where $\epsilon_{t}$ is i.i.d. $N\left(0, \omega^{2}\right)$. It is easy to see that in this case $Z_{m, i} \stackrel{d}{=} \mu_{m, i} \cdot \chi^{2}(1)$ where $\chi^{2}(1)$ denotes the standard chi-squared distribution with 1 degree of freedom. Whereas $B_{m, i} \stackrel{d}{=}\left(\frac{\psi_{1}^{k_{n}} d_{n}}{2 k_{n}^{2} \psi_{2}^{k_{n}}} \omega^{2}\right) \cdot \sum_{i=1}^{k_{n}-1} \widetilde{U}_{i}^{2}$, where $\left(\widetilde{U}_{i}\right)_{i=1}^{k_{n}-1}$ are one-dependent standard normal random variables with $\operatorname{Cov}\left(\widetilde{U}_{i}, \widetilde{U}_{i-1}\right)=-1$. In addition, $Z_{m, i}$ and $B_{m, i}$ are dependent. Thus, in this relatively simple context one could ensure the validity of the Cramer's condition by showing that $Z_{m, i}-B_{m, i}$ have a nonlattice distribution, something we have not attempted to prove in this paper. In presence of noise, it would clearly be desirable to have a formal proof of the verification of Cramer's condition, but this is beyond the scope of this paper. In this section, our approach is similar to those used, e.g., by Mammen (1993), Davidson and Flachaire (2001) and GM (2009). Our main focus is on using formal Edgeworth expansions to explain the superior finite sample properties of the wild bootstrap procedure applied on the non-overlapping pre-averaged returns as recently studied by Gonçalves et al. (2014). Note however that in contrast to GM (2009) (under no noise), we explicitly provide (high level) sufficient conditions that ensure the validity of our Edgeworth expansions in the noisy setting.

Corollary 3.1. Let $T_{n}$ and $\widetilde{T}_{n}$ be defined as

$$
T_{n}=\frac{n^{1 / 4}\left(\widehat{\Gamma}_{n}-\Gamma\right)}{\sqrt{\hat{V}_{n}}} \text { and } \widetilde{T}_{n}=\frac{n^{1 / 4}\left(\widehat{\Gamma}_{n}+\widetilde{b}_{n}-(\Gamma+\widetilde{b})\right)}{\sqrt{\hat{V}_{n}}}
$$


where $\widehat{\Gamma}_{n}$ and $\hat{V}_{n}$ are given by (16) and (18), respectively and $\widetilde{b}_{n}=\frac{\psi_{1}^{k_{n}}}{2 k_{n}^{2} \psi_{2}^{k_{n}}} \sum_{i=1}^{n}\left(\Delta_{i}^{n} Y\right)^{2}, \widetilde{b}=\frac{\psi_{1}}{\theta^{2} \psi_{2}} \omega^{2}$. Suppose (1) holds with $b=0$. Under Assumptions 1, 3, 4 and 5, and conditionally on $\sigma$, the formal second-order Edgeworth expansions of the studentized statistics $T_{n}$ and $\widetilde{T}_{n}$ are exactly the same.

Remark 5. The bias term in the pre-averaging estimator does not impact the second-order Edgeworth expansion. Here, we provide the main idea behind this via a toy example involving normalized i.i.d. statistics. Let $\left(M_{n, i}\right)_{i=1}^{n},\left(N_{n, i}\right)_{i=1}^{n}$ be two triangular arrays of row-wise i.i.d. random variables with mean zero and order $O(1)$. Let $\sigma_{n}^{2}=\mathbb{E}\left[M_{n, 1}^{2}\right]$ and $\mu_{n, 3}=\mathbb{E}\left[M_{n, 1}^{3}\right]$. Define

$$
S_{n}=\frac{1}{\sigma_{n} \sqrt{n}} \sum_{i=1}^{n} M_{n, i}, \text { and } U_{n}=\frac{1}{\sigma_{n} \sqrt{n}} \sum_{i=1}^{n}\left(M_{n, i}+\frac{1}{\sqrt{n}} N_{n, i}\right) .
$$

It is well-known that, under the existence of third moments and Cramer's condition, the second-order Edgeworth expansion of $S_{n}$ is

$$
\Phi(x)+\frac{1}{\sqrt{n}} \frac{\mu_{n, 3}}{6 \sigma_{n}^{3}} \phi(x),
$$

where $\Phi(x)$ and $\phi(x)$ are the distribution and the density functions of the standard normal. It turns out that the term $U_{n}$ has also the same Edgeworth expansion if $M_{n, i}$ and $N_{n, i}$ are "weakly" correlated. Let's assume that $\mathbb{E}\left[M_{n, 1} N_{n, 1}\right]=O\left(n^{-1 / 2}\right)$. Then

$$
s_{n}^{2} \equiv \operatorname{Var}\left(M_{n, 1}+n^{-1 / 2} N_{n, 1}\right)=\sigma_{n}^{2}+2 n^{-1 / 2} \mathbb{E}\left[M_{n, 1} N_{n, 1}\right]+n^{-1} \mathbb{E}\left[N_{n, 1}^{2}\right]=\sigma_{n}^{2}+O\left(n^{-1}\right) .
$$

Now, we decompose $U_{n}$ as

$$
U_{n}=\frac{1}{s_{n} \sqrt{n}} \sum_{i=1}^{n}\left(M_{n, i}+\frac{1}{\sqrt{n}} N_{n, i}\right)+\left(\frac{1}{\sigma_{n} \sqrt{n}}-\frac{1}{s_{n} \sqrt{n}}\right) \sum_{i=1}^{n}\left(M_{n, i}+\frac{1}{\sqrt{n}} N_{n, i}\right) \equiv \hat{U}_{n}+R_{n} .
$$

We note that the term $R_{n}$ does not contribute to the second-order Edgeworth expansion of $U_{n}$ due to (33). And $\hat{U}_{n}$ has the same form as $S_{n}$ (i.e., a normalized statistic) and hence possesses the same second-order Edgeworth expansion in view of

$$
\mathbb{E}\left[\left(M_{n, 1}+n^{-1 / 2} N_{n, 1}\right)^{3}\right]=\mu_{n, 3}+O\left(n^{-1 / 2}\right) \text { and } s_{n}^{3}=\sigma_{n}^{3}+O\left(n^{-1 / 2}\right) .
$$

Proposition 3.2. Let $T_{n}$ and $T_{n}^{*}$ be defined as in (17) and (19), respectively. Suppose that $v_{i}$ has the same distribution as in Proposition 3.1 and (1) holds with $b=0$. Under Assumptions 1, 3, 4 and 5, conditionally on $\sigma$, as $n \rightarrow \infty$, we get

$$
\sup _{x \in \mathbb{R}}\left|\mathbb{P}^{*}\left[T_{n}^{*} \leq x\right]-\mathbb{P}\left[T_{n} \leq x\right]\right|=o_{p}\left(n^{-1 / 4}\right) .
$$

Proposition 3.2 shows the second-order validity of the wild bootstrap method in the noisy setting and hence extends the result obtained in Proposition 3.1.

\subsection{Edgeworth corrected interval for realized volatility estimators}

Our aim in this section is to explain how one can use the Edgeworth expansions derived in Sections 3.1 and 3.2 to construct valid confidence intervals for integrated volatility with improved coverage probabilities. Our approach follows Hall (1992), see also GM (2008). In particular, based on Edgeworth expansions of $\widehat{\Gamma}_{n}$, we define confidence intervals for $\Gamma$ corrected by these Edgeworth expansions. Here, we consider one-sided Edgeworth expansion corrected intervals for $\Gamma$. 
One can show that (see, e.g., Podolskij and Vetter (2009)), as $n \rightarrow \infty$

$$
\widetilde{R}_{r, s} \stackrel{\mathbb{P}}{\rightarrow} a_{r, s} \widetilde{\sigma}_{r, s}
$$

Thus, when the log price process follows (1) with $b=0$, we propose the following feasible (empirical) version of $q_{1}(x)$,

$$
\hat{q}_{1}(x)=\frac{4\left(2 x^{2}+1\right) a_{6,4}^{-1} \widetilde{R}_{6,4}}{6 \sqrt{2}} .
$$

A one-sided feasible Edgeworth expansion corrected 100(1- $\alpha) \%$ level interval for $\Gamma$ is given by:

$$
I C_{\text {feas }, 1-\alpha}^{E E-1}=\left(-\infty, \widehat{\Gamma}_{n}-\tau_{n}^{-1} \sqrt{\hat{V}_{n}} z_{\alpha}+\tau_{n}^{-2} \sqrt{\hat{V}_{n}} \hat{q}_{1}\left(z_{\alpha}\right)\right) .
$$

In contrast to the conventional intervals based on the normal approximation, this interval contains a skewness correction term equal to $\tau_{n}^{-2} \sqrt{\hat{V}_{n}} \hat{q}_{1}\left(z_{\alpha}\right)$. Here, we do not pursue the derivation of a two-sided symmetric feasible Edgeworth expansion corrected 100(1- $\alpha) \%$ level interval for $\Gamma$. The main reason is because for this interval, in contrast to $I C_{f e a s, 1-\alpha}^{E E-1}$ would involve in addition to a skewness term a kurtosis correction term which is not available under results derived in Theorems 3.1 and $3.2 .^{2}$

Remark 6. Our setting rules out leverage effects, which is the case when $\sigma$ and $W$ are correlated. Indeed, under no leverage assumptions, it is possible for us to condition on the path of $\sigma$ and then use the independence of increments. However, if $\sigma$ and $W$ are correlated, we only have a martingale difference sequence instead of the independence property and hence this approach breaks down. Recent work of Yoshida (2013) develops a general theory to deal with Edgeworth expansions involving mixed normal limits. We note that this work relies on very technical tools from Malliavin calculus which are beyond the scope of this paper. Podolskij and Yoshida (2014) apply this theory within the framework of power variations of diffusion processes. Although the last work allows leverage effects, it is assumed that $\sigma$ is driven (only) by the original Brownian motion $W$, thereby excluding stochastic volatility models. While these works are limited to the setting of continuous volatility, our setting allows (in particular, in the no noise case) discontinuous volatility paths.

\section{Monte Carlo simulations}

Our aim here is to compare the finite sample performance of the Edgeworth expansion corrected intervals in comparison to the feasible asymptotic theory-based intervals and the bootstrap method of Gonçalves et al. (2014) using noisy diffusion model. The design of our Monte Carlo study is roughly identical to that used by Gonçalves et al. (2014) with some minor differences. In particular, we only consider the two-factor stochastic volatility (SV2F) model analyzed by Gonçalves et al. (2014) since it is more empirically relevant and exhibits overall larger coverage distortions than the one-factor stochastic volatility model. Here we briefly describe the Monte Carlo design we use.

\footnotetext{
${ }^{2}$ We refer to Hall (1992), GM (2008) and Zhang et al. (2011) for further details that explain why these intervals are expected to outperform the conventional intervals based on the normal approximation. In the context of no noise, GM (2008) also derived a two-sided symmetric feasible Edgeworth expansion corrected interval for $\Gamma$.
} 
To simulate log-prices we consider the following SV2F model, where ${ }^{3}$

$$
\begin{aligned}
d X_{t} & =b d t+\sigma_{t} d W_{t}, \\
\sigma_{t} & =s-\exp \left(\beta_{0}+\beta_{1} \tau_{1 t}+\beta_{2} \tau_{2 t}\right), \\
d \tau_{1 t} & =\widetilde{\alpha}_{1} \tau_{1 t} d t+d B_{1 t}, \\
d \tau_{2 t} & =\widetilde{\alpha}_{2} \tau_{2 t} d t+\left(1+\phi \tau_{2 t}\right) d B_{2 t}, \\
\operatorname{corr}\left(d W_{t}, d B_{1 t}\right) & =\varphi_{1}, \operatorname{corr}\left(d W_{t}, d B_{2 t}\right)=\varphi_{2} .
\end{aligned}
$$

Our baseline model sets $b=0$ and $\varphi_{1}=\varphi_{2}=0$ which is compatible with the assumption of no leverage and no drift. While the theory of the Edgeworth expansion developed in this paper does not allow the leverage effect, we have also studied this setup which is nevertheless an obvious interest in practice and set $b=0.03$ and $\varphi_{1}=\varphi_{2}=-0.3$. In both cases, we follow Huang and Tauchen (2005) and set $\beta_{0}=-1.2, \beta_{1}=0.04, \beta_{2}=1.5, \widetilde{\alpha}_{1}=-0.00137, \widetilde{\alpha}_{2}=-1.386$, and $\phi=0.25$. We initialize the two factors at the start of each interval by drawing the persistent factor from its unconditional distribution, $\tau_{10} \sim N\left(0, \frac{-1}{2 \widetilde{\alpha}_{1}}\right)$, and by starting the stronlgly mean-reverting factor at zero. For $i=1, \ldots, n$, we let the market microstructure noise be defined as $\epsilon_{\frac{i}{n}} \sim$ i.i.d. $N\left(0, \omega^{2}\right)$. The size of the noise is an important parameter. We follow Barndorff-Nielsen et al. (2008) and model the noise magnitude as $\xi^{2}=\omega^{2} / \sqrt{\int_{0}^{1} \sigma_{s}^{4} d s}$. We fix $\xi^{2}$ equal to $0.0001,0.001$ and 0.01 and let $\omega^{2}=\xi^{2} \sqrt{\int_{0}^{1} \sigma_{s}^{4} d s}$. These values are motivated by the empirical study of Hansen and Lunde (2006), who investigate 30 stocks of the Dow Jones Industrial Average.

We simulate data for the unit interval $[0,1]$ and normalize one second to be $1 / 23400$, so that $[0,1]$ is thought to span 6.5 hours. The observed $Y$ process is generated using an Euler scheme. We then construct the $\frac{1}{n}$-horizon returns $\Delta_{i}^{n} Y \equiv Y_{i / n}-Y_{(i-1) / n}$ based on samples of size $n$.

The pre-averaging approach requires the choice of the window length $k_{n}=\theta \sqrt{n}$ over which the pre-averaging of returns is done. In our simulations, we follow Christensen et al. (2010) and use a conservative choice of $k_{n}$ (this corresponds to let $\theta=1$ ). We also follow the literature and use the weight function $g(x)=\min (x, 1-x)$ to compute the pre-averaged returns. In order to reduce finite sample biases associated with Riemann integrals, we follow Jacod et al. (2009) and Hautsch and Podolskij (2013) and use the finite sample adjustments version of the pre-averaged realized volatility estimator,

$$
\widehat{\Gamma}_{n}=\left(1-\frac{\psi_{1}^{k_{n}}}{2 n \theta^{2} \psi_{2}^{k_{n}}}\right)^{-1}\left(\frac{1}{\psi_{2}^{k_{n}}} \sum_{m=0}^{d_{n}-1} \bar{Y}_{m k_{n}}^{2}-\frac{\psi_{1}^{k_{n}}}{2 k_{n}^{2} \psi_{2}^{k_{n}}} \sum_{i=1}^{n}\left(\Delta_{i}^{n} Y\right)^{2}\right)
$$

where $\psi_{1}^{k_{n}}=k_{n} \sum_{i=1}^{k_{n}}\left(g\left(\frac{i}{k_{n}}\right)-g\left(\frac{i-1}{k_{n}}\right)\right)^{2}$ and $\psi_{2}^{k_{n}}=\frac{1}{k_{n}} \sum_{i=1}^{k_{n}} g^{2}\left(\frac{i}{k_{n}}\right)$.

Tables 1 gives the actual rates of $95 \%$ one-sided confidence intervals of integrated volatility for the SV2F model, computed over 10,000 replications. Results are presented for eight different samples sizes: $n=23400,11700,7800,4680,1560,780,390$ and 195, corresponding to "1-second", "2-second", "3-second", "5-second", "15-second", "30-second", "1-minute" and "2-minute" frequencies. In our simulations, bootstrap intervals use 999 bootstrap replications for each of the 10,000 Monte Carlo replications. We consider one-sided bootstrap percentile- $t$ interval computed at the $95 \%$ level given by (11). To generate the bootstrap data we use two different external random variables.

WB1 The two point distribution initially proposed by GM (2009), where $v_{j} \sim$ i.i.d. such that:

$$
v_{j}=\left\{\begin{array}{l}
\frac{1}{5} \sqrt{31+\sqrt{186}}, \text { with probability } p=\frac{1}{2}-\frac{3}{\sqrt{186}} \\
-\frac{1}{5} \sqrt{31-\sqrt{186}}, \text { with probability } 1-p
\end{array},\right.
$$

\footnotetext{
${ }^{3}$ The function s-exp is the usual exponential function with a linear growth function splined in at high values of its argument: $s-\exp (x)=\exp (x)$ if $x \leq x_{0}$ and $s-\exp (x)=\frac{\exp \left(x_{0}\right)}{x_{0}} \sqrt{x_{0}-x_{0}^{2}+x^{2}}$ if $x>x_{o}$, with $x_{0}=\log (1.5)$.
} 
for which we have $\mu_{2}^{*}=1$ and $\mu_{4}^{*}=31 / 25$.

WB2 A two point distribution $v_{j} \sim$ i.i.d. such that:

$$
v_{j}=\left\{\begin{array}{llll}
\left(\frac{2}{3}\right)^{1 / 4} \frac{-1+\sqrt{5}}{2}, & \text { with } & \text { probability } & p=\frac{\sqrt{5}-1}{2 \sqrt{5}} \\
\left(\frac{2}{3}\right)^{1 / 4} \frac{-1-\sqrt{5}}{2}, & \text { with } & \text { probability } & 1-p=\frac{\sqrt{5}+1}{2 \sqrt{5}}
\end{array},\right.
$$

for which $\mu_{2}^{*}=2 \sqrt{2 / 3}$ and $\mu_{4}^{*}=10 / 3$.

WB3 The new optimal nonlattice distributiont $v_{j} \sim$ i.i.d. with the same distribution as in Proposition 3.1.

Note that all of these choices of $v_{j}$ are asymptotically valid when used to construct bootstrap percentile- $t$ intervals. As we formally show in this paper, the choice of WB3 is still optimal to provide a second-order asymptotic refinement for the wild bootstrap method applied on the non-overlapping pre-averaged returns. The wild bootstrap based on WB1 is able to match the first and third cumulants of pre-averaged realized volatility, but as a lattice distribution may not satisfy the Cramer's condition. Based on simulations results, Gonçalves et al. (2014) advocated the use of WB2. In Table 1, "CLT" refers to the value predicted by the normal asymptotic, "EE-est" refers to the value based on Edgeworth expansion corrected intervals, whereas "WB1", "WB2" and "WB3" refer to the value predicted by the bootstrap method based on external random variable WB1, WB2 and WB3, respectively.

Starting with the baseline model: no leverage and no drift, an inspection of Table 1 suggests that all intervals tend to undercover. The degree of undercoverage is especially large for smaller values of $n$, when sampling is not too frequent. Results seem to be not very sensitive to the noise magnitude. Onesided confidence intervals based on the asymptotic normal theory (without higher order correction) is not adequate to capture the skewness in the $t$ statistics (as confirmed by simulations not reported here). Hounyo et al. (2014) (cf. Session 3) also found similar pattern for symmetric two-sided confidence intervals. See Hounyo et al. (2014) for more results on the comparison between this model from the viewpoint of skewness and kurtosis.

Overall, the WB2 does very well for small samples $(n=195,390$ and 780$)$ whereas WB1 and WB3-based intervals do very well for large samples $(n=11700$ and 23400$)$. For instance, when $\xi^{2}=0.0001$, WB2 has a coverage probability equal to $86.87 \%$ when $n=195$, whereas WB1 and WB3 cover integrated volatility only $77.56 \%$ and $80.06 \%$ of the time, respectively. These rates increase to $93.59 \%, 95.90 \%$ and $92.41 \%$, respectively, for $n=23400$. Results also confirm that, our expansion theory provides a good approximation of the small sample distribution of Podolskij and Vetter's (2009) pre-averaged realized volatility estimator. In particular, for all sample sizes consider here, the intervals based on the Edgeworth corrections (EE-est) have improved properties relatively to the conventional intervals based on the normal approximation. Contrary to the bootstrap, the Edgeworth approach is an analytical approach that is easily implemented, without requiring any resampling of one's data. A comparison between the bootstrap (WB1, WB2 and WB3) and the Edgeworth expansion shows that the bootstrap outperforms the Edgeworth corrected intervals. For instance, when $\xi^{2}=0.001$, and we resample every 5 -second $(n=4680)$, the CLT-based interval has a coverage probability equal to $84.82 \%$, whereas EE-est based interval covers integrated volatility $89.51 \%$ of the time. For the bootstrap, these rates increase to $93.59 \%, 92.27 \%$ and $90.09 \%$ for WB1, WB2 and WB3, respectively.

Notice, however that results based on WB1 and WB3 intervals are close, but slightly different especially for small samples $(n=195,390$ and 780$)$. This observation suggests that, the dominance of WB1 by WB2 for $\mathrm{n}$ small is not due to the possibly non invalidity of the Edgeworth expansions for realized volatility based on WB1, i.e. the optimal two-point distribution wild bootstrap. The good performance of WB2 over WB1 and WB3 in smaller sample size is similar to the superior performance 
of the i.i.d. bootstrap over the optimal two-point distribution WB1 in GM (2009). Indeed, Monte Carlo simulations in GM (2009) show that despite the fact that the i.i.d. bootstrap does not theoretically provide an asymptotic refinement for one-sided confidence intervals when the volatility is stochastic, this latter outperforms WB1. Accordingly, it would be useful to develop a new theory that provides a more reliable guide to gauge the finite sample performance of the bootstrap for financial high-frequency data.

A similar pattern is observed for all intervals in presence of drift and leverage effects. For all methods, results are robust to drift and leverage effects. In particular, despite the fact that our Edgeworth expansion corrected intervals do not theoretically take into account these effects, EE-est outperform the CLT-based intervals in presence of drift and leverage effects. 
Table 1. Coverage rate of nominal $95 \%$

\begin{tabular}{|c|c|c|c|c|c|c|c|c|c|c|}
\hline \multirow[t]{2}{*}{$\bar{n}$} & \multicolumn{5}{|c|}{ No Leverage and No Drift } & \multicolumn{5}{|c|}{ With Leverage and Drift } \\
\hline & CLT & EE-est & WB1 & WB2 & WB3 & CLT & EE-est & WB1 & WB2 & WB3 \\
\hline \multicolumn{11}{|c|}{$\xi^{2}=0.0001$} \\
\hline 195 & 67.98 & 76.32 & 77.56 & 86.87 & 80.06 & 68.77 & 76.23 & 77.98 & 86.84 & 80.45 \\
\hline 390 & 76.07 & 82.83 & 85.71 & 90.01 & 85.17 & 76.01 & 83.05 & 86.16 & 90.24 & 85.50 \\
\hline 780 & 78.44 & 85.11 & 88.65 & 90.64 & 86.13 & 77.98 & 84.51 & 88.22 & 90.33 & 87.10 \\
\hline 1560 & 83.21 & 88.72 & 92.32 & 92.42 & 89.56 & 83.63 & 88.78 & 92.54 & 92.52 & 89.57 \\
\hline 4680 & 84.73 & 89.37 & 93.37 & 92.19 & 90.20 & 84.65 & 89.47 & 93.50 & 92.46 & 90.41 \\
\hline 7800 & 86.31 & 90.60 & 94.42 & 93.05 & 91.29 & 86.32 & 90.63 & 94.47 & 93.06 & 91.57 \\
\hline 11700 & 87.07 & 91.44 & 95.17 & 93.47 & 91.87 & 87.70 & 91.69 & 95.10 & 93.79 & 92.05 \\
\hline 23400 & 88.26 & 91.86 & 95.90 & 93.59 & 92.41 & 88.43 & 92.04 & 95.85 & 93.80 & 92.16 \\
\hline
\end{tabular}

\begin{tabular}{lllllllllll}
\multicolumn{2}{l}{$\xi^{2}=0.001$} \\
\hline 195 & 68.20 & 76.71 & 78.13 & 86.98 & 80.42 & 68.76 & 76.60 & 78.67 & 87.08 & 80.42 \\
390 & 76.21 & 83.20 & 85.86 & 89.88 & 85.36 & 76.06 & 83.40 & 86.19 & 90.11 & 85.58 \\
780 & 78.71 & 85.19 & 88.85 & 90.69 & 86.16 & 77.86 & 84.59 & 88.30 & 90.28 & 86.97 \\
1560 & 83.39 & 88.67 & 92.24 & 92.34 & 89.65 & 83.55 & 88.82 & 92.53 & 92.75 & 89.85 \\
4680 & 84.82 & 89.51 & 93.59 & 92.27 & 90.09 & 84.69 & 89.77 & 93.58 & 92.65 & 90.36 \\
7800 & 86.38 & 90.93 & 94.37 & 93.16 & 91.25 & 86.31 & 90.66 & 94.46 & 93.10 & 91.76 \\
11700 & 87.16 & 91.39 & 95.10 & 93.32 & 91.94 & 87.66 & 91.65 & 95.10 & 93.79 & 92.15 \\
23400 & 88.40 & 91.89 & 95.70 & 93.58 & 92.36 & 88.40 & 91.89 & 95.80 & 93.82 & 92.10
\end{tabular}

\begin{tabular}{lllllllllll}
\multicolumn{2}{l}{$\xi^{2}=0.01$} \\
\hline 195 & 70.55 & 78.70 & 81.07 & 87.61 & 81.11 & 70.21 & 78.66 & 80.65 & 87.09 & 81.25 \\
390 & 77.63 & 84.36 & 87.64 & 90.46 & 86.24 & 77.35 & 84.14 & 87.88 & 90.42 & 86.65 \\
780 & 79.84 & 86.12 & 89.98 & 91.16 & 86.56 & 79.21 & 85.22 & 89.29 & 90.13 & 87.13 \\
1560 & 84.09 & 89.24 & 92.98 & 92.49 & 90.48 & 84.00 & 89.33 & 93.15 & 92.54 & 90.45 \\
4680 & 85.31 & 90.18 & 94.18 & 92.87 & 90.54 & 85.43 & 90.20 & 94.35 & 93.02 & 90.88 \\
7800 & 86.82 & 91.02 & 94.88 & 93.23 & 91.53 & 86.81 & 90.60 & 94.56 & 92.94 & 91.59 \\
11700 & 87.59 & 91.35 & 95.23 & 93.35 & 92.20 & 88.05 & 91.72 & 95.05 & 93.50 & 92.38 \\
23400 & 88.76 & 92.05 & 95.87 & 93.53 & 92.86 & 89.01 & 92.47 & 95.95 & 94.02 & 92.43 \\
\hline \hline
\end{tabular}

Notes: CLT-intervals based on the Normal; EE-est refers to the value based on Edgeworth expansion corrected intervals; WB1 wild bootstrap intervals based on the external random variable WB1; WB2 wild bootstrap intervals based on the external random variable WB2; WB3 wild bootstrap intervals based on the external random variable WB2. Ten thousand Monte Carlo trials with 999 bootstrap replications each. 


\section{Conclusion}

The main contribution of this paper has been to establish the theoretical validity of the Edgeworth expansions for realized volatility estimators. Furthermore, we propose a new optimal nonlattice distribution for the wild bootstrap suggested by GM (2009) is able to provide a second-order asymptotic refinement. In the presence of microstructure noise, based on our Edgeworth expansions, we show that the new optimal choice proposed in the absence of noise is still valid in noisy data for the pre-averaged realized volatility estimator proposed by Podolskij and Vetter (2009). Finally, we also propose confidence intervals for integrated volatility that incorporate an analytical correction for skewness as alternative method of inference. Thus, we extend existing results in GM (2008) by allowing for microstructure noise. The results of our Monte Carlo study show that the Edgeworth-based coverage probabilities provide very accurate approximations to the sample ones, compared to the normal based coverage probabilities. A comparison between the bootstrap and the Edgeworth expansion shows that the bootstrap-based intervals outperform the Edgeworth corrected intervals.

In the process of developing the expansions for realized volatility estimators, we also shows how to derive the second-order Edgeworth expansions of a certain form of studentized statistic $t$, where observations are independent but not identically distributed with a specific heterogeneity properties (Proposition 6.1 in the Appendix). This result should have applications to other situations.

Establishing the validity of the Edgeworth expansions for realized volatility estimators under general conditions which allow drift and leverage effects as for instance in Barndorff-Nielsen, Graversen, Jacod, and Shephard (2006) is a promising extension of this work. Another important extension is to prove similar results for others existing noise and/or jump robust realized volatility measures. These extensions are left for future research.

\section{Appendix: proofs of the validity of Edgeworth expansion}

\subsection{Auxiliary results}

The main goal of this section is to prove Proposition 6.1. In Section 6.2, we will show that the main results of this paper belong to the framework of Proposition 6.1.

In this section, we deal with 2 -dimensional random vectors. We denote transposes by ' and for $x=\left(x_{1}, x_{2}\right)^{\prime} \in \mathbb{R}^{2}$ and $\nu=\left(\nu_{1}, \nu_{2}\right)^{\prime} \in \mathbb{N}^{2}$ we use the notations

$$
\|x\|=\sqrt{x_{1}^{2}+x_{2}^{2}} \text { and } x^{\nu}=\left(x_{1}\right)^{\nu_{1}}\left(x_{2}\right)^{\nu_{2}} .
$$

For the mean zero triangular array $\left(A_{m, i}\right), m \geq 1,1 \leq i \leq m$ and $p \geq 2$, we denote

$$
\rho_{m, p}=m^{-1} \sum_{i=1}^{m} E\left[\left\|A_{m, i}\right\|^{p}\right] .
$$

Below we provide sufficient conditions for the validity of the Edgeworth expansion.

Assumption 6. Let $\left(A_{m, i}\right)_{i=1}^{m}, m \geq 1$, be row-wise independent triangular array of 2-dimensional random vectors with mean zero such that

(i) For all $m \geq 1$, we have

$$
\frac{1}{m} \sum_{i=1}^{m} \mathbb{E}\left[A_{m, i} A_{m, i}^{\prime}\right]=\mathbb{I}_{2} .
$$

(ii) There exists $\delta>0$ and $C>0$ such that $\mathbb{E}\left[\left\|A_{m, i}\right\|^{3+\delta}\right] \leq C$ for all $i, m$. 
(iii) There exists $M \in(0,1)$ such that

$$
\left|\phi_{m, i}(t)\right| \leq M \text { for }\|t\| \geq\left(16 \rho_{m, 3}\right)^{-1} \text { and } m \geq 1,1 \leq i \leq m,
$$

where $\phi_{m, i}$ is the characteristic function of $A_{m, i}$.

We note that some initial Hermite polynomials are given by

$$
H_{0}(x)=1, H_{1}(x)=x, H_{2}(x)=x^{2}-1, H_{3}(x)=x^{3}-3 x .
$$

For $1 \leq i \leq m$, and $\nu \in \mathbb{N}^{2}$ we define

$$
\chi_{\nu, i}=\mathbb{E}\left[\left(A_{m, i}\right)^{\nu}\right] \text { and } \bar{\chi}_{\nu}^{m}=m^{-1} \sum_{i=1}^{m} \chi_{\nu, i} .
$$

We recall that the 2-dimensional polynomial appearing in the second-order Edgeworth expansion is given by (see Section 7 in Bhattacharya and Rao (1986))

$$
p_{1}(t, s)=\sum_{j=0}^{3} \frac{\bar{\chi}_{(3-j, j)}^{m} H_{3-j}(t) H_{j}(s)}{(3-j) ! j !} .
$$

Now, we are ready to state the following classical result on Edgeworth expansions.

Lemma 6.1. Suppose that the 2-dimensional random vectors $\left(A_{m, i}\right)_{i=1}^{m}$ satisfy Assumption 6 . Let

$$
S_{m}=\frac{1}{\sqrt{m}} \sum_{i=1}^{m} A_{m, i}
$$

Then, the second-order Edgeworth expansion of $S_{m}$ is given by

$$
\mathbb{P}\left[S_{m} \in(-\infty, y] \times(-\infty, z]\right]=\int_{-\infty}^{y} \int_{-\infty}^{z}\left(1+m^{-1 / 2} p_{1}(t, s)\right) \phi(s, t) d s d t+o\left(m^{-1 / 2}\right)
$$

uniformly in $x$.

Proof. The result follows from Theorem 6.2 in Lahiri (2003) as it is easy to show that Assumption 6 satisfies the conditions of Theorem 6.2 in Lahiri (2003).

Now, we are ready to describe the form of the $t$-statistic. We define

$$
t_{m}=\frac{\frac{1}{\sqrt{m}} \sum_{i=1}^{m}\left(Z_{m, i}-\mu_{m, i}-B_{m, i}\right)+\frac{1}{\sqrt{m}} b_{m}}{\sqrt{V_{m}}}
$$

with

$$
V_{m}=\frac{a_{4}-a_{2}^{2}}{a_{4}} \frac{1}{m} \sum_{i=1}^{m} Z_{m, i}^{2}
$$

where the structure of $Z_{m, i}, \mu_{m, i}, B_{m, i}, b_{m}$ and $a_{s}$ are provided below. To prove the Edgeworth expansion for $t_{m}$, we need the following conditions.

Assumption 7. We suppose that $t_{m}$ in (37) satisfies 
(i) For each $m \geq 1$, the random vectors $\left(Z_{m, i}, B_{m, i}\right)_{i=1}^{m}$ are independent and $Z_{m, i}$ has the representation $Z_{m, i}=\left(\alpha_{m, i} u_{m, i}+\beta_{m, i}\right)^{2}$, where $\alpha_{m, i}$ and $\beta_{m, i}$ are real triangular arrays and $u_{m, i} \sim U$ for all $i, m$. We denote $a_{s}=\mathbb{E}\left[|U|^{s}\right]$ and impose $a_{2}>0$. In addition, we have $\mathbb{E}\left[B_{m, i}\right]=0$ for all $i, m$.

(ii) Let's denote $\mu_{m, i} \equiv \mathbb{E}\left[Z_{m, i}\right]$. There exists $C>0$ such that for all $i$ and $m$, we obtain

$$
\left|\mathbb{E}\left[Z_{m, i}^{2}\right]-\frac{a_{4}}{a_{2}^{2}} \mu_{m, i}^{2}\right|+\left|\mathbb{E}\left[Z_{m, i}^{3}\right]-\frac{a_{6}}{a_{2}^{3}} \mu_{m, i}^{3}\right|+\left|\mathbb{E}\left[Z_{m, i} B_{m, i}\right]\right|+\left|\mathbb{E}\left[Z_{m, i}^{2} B_{m, i}\right]\right| \leq \frac{C}{m}
$$

and

$$
\mathbb{E}\left[\left|Z_{m, i}\right|^{2(3+\delta)}\right]+\mathbb{E}\left[\left|\sqrt{m} B_{m, i}\right|^{3+\delta}\right] \leq C .
$$

(iii) For all $r>0$, there exists $M_{r} \in(0,1)$ such that

$$
\left|\phi_{m, i}(t)\right| \leq M_{r} \text { for all }\|t\| \geq r \text { and } m \geq 1,1 \leq i \leq m,
$$

where $\phi_{m, i}$ is the characteristic function of $\left(Z_{m, i}-\mu_{m, i}-B_{m, i}, Z_{m, i}^{2}-\mathbb{E}\left[Z_{m, i}^{2}\right]\right)^{\prime}$.

(iv) There exists $\nu>0$ such that for all $m \geq 1$ we have

$$
\nu \leq \frac{1}{m} \sum_{i=1}^{m} \mathbb{E}\left[Z_{m, i}^{2}\right] \text { and } \nu \leq \min \left(v_{m}^{2}, w_{m}^{2}-u_{m}^{2}\right)
$$

where

$$
\begin{aligned}
& v_{m}^{2}=\frac{1}{m} \sum_{i=1}^{m} \operatorname{Var}\left(Z_{m, i}-\mu_{m, i}-B_{m, i}\right), \quad E_{m}=\frac{1}{\sqrt{m} v_{m}} \sum_{i=1}^{m}\left(Z_{m, i}-\mu_{m, i}-B_{m, i}\right) \\
& F_{m}=\frac{a_{4}-a_{2}^{2}}{a_{4}} \frac{1}{\sqrt{m}} \sum_{i=1}^{m}\left(Z_{m, i}^{2}-\mathbb{E}\left[Z_{m, i}^{2}\right]\right), \quad u_{m}=\operatorname{Cov}\left(E_{m}, F_{m}\right), \quad w_{m}^{2}=\operatorname{Var}\left(F_{m}\right) .
\end{aligned}
$$

(v) There exists $\hat{b} \in \mathbb{R}$ and $C>0$ such that the real sequence $\left(b_{m}\right)_{m \geq 1}$ satisfies

$$
\left|\frac{b_{m}}{v_{m}}-\hat{b}\right| \leq \frac{C}{\sqrt{m}}
$$

Note that when $u_{m, i} \sim N(0,1)$, first few even moments of $u_{m, i}$ are given by $a_{2}=1, a_{4}=3$ and $a_{6}=15$. We will also use analogous results with the proposition below for the bootstrap, which may have different moments $a_{s}$.

Before we state the main result we need a final notation. For each $p \geq 1$, we denote

$$
\kappa_{m, p}=\frac{1}{m} \sum_{i=1}^{m}\left(\mu_{m, i}\right)^{p} .
$$

Proposition 6.1. Under Assumption 7, we obtain

$$
\mathbb{P}\left[t_{m} \leq x\right]=\Phi(x)+m^{-1 / 2}\left[\left(\frac{A_{1}}{2}-\frac{1}{6}\left(B_{1}-3 A_{1}\right)\left(x^{2}-1\right)\right) \frac{\kappa_{m, 3}}{\left(\kappa_{m, 2}\right)^{3 / 2}}-\hat{b}\right] \phi(x)+o\left(m^{-1 / 2}\right)
$$

uniformly in $x$, where

$$
A_{1}=\frac{a_{6}-a_{2} a_{4}}{a_{4}\left(a_{4}-a_{2}^{2}\right)^{1 / 2}} \text { and } B_{1}=\frac{a_{6}-3 a_{2} a_{4}+2 a_{2}^{3}}{\left(a_{4}-a_{2}^{2}\right)^{3 / 2}} .
$$


Proof. It will be convenient to write the studentized statistic in the following way:

$$
t_{m}=\frac{E_{m}+m^{-1 / 2} b_{m} / v_{m}}{\sqrt{V_{m} / v_{m}^{2}}} \equiv \frac{\widetilde{E}_{m}}{\sqrt{V_{m} / v_{m}^{2}}} .
$$

Using Taylor's series for $f(x)=x^{-1 / 2}$ of $V_{m} / v_{m}^{2}$ around 1 , we obtain

$$
\frac{1}{\sqrt{V_{m} / v_{m}^{2}}}=1-\frac{V_{m}-v_{m}^{2}}{2 v_{m}^{2}}+\frac{3}{8\left(\xi_{m}\right)^{5 / 2}} \frac{\left(V_{m}-v_{m}^{2}\right)^{2}}{v_{m}^{4}}
$$

where $\xi_{m}$ is between 1 and $V_{m} / v_{m}^{2}$. Next, we observe that Assumption 7(ii) implies

$$
\left|\left(V_{m}-v_{m}^{2}\right)-\frac{1}{\sqrt{m}} F_{m}\right| \leq \frac{C}{m}
$$

for some $C>0$. Using above identities and Assumption 7(v), we may decompose:

$$
t_{m}=U_{m}+m^{-1 / 2} \hat{b}+R_{m}
$$

where the leading term is

$$
U_{m}=E_{m}-\frac{1}{2 \sqrt{m}} \frac{E_{m} F_{m}}{v_{m}^{2}}
$$

whereas the remainder term is given by

$$
\begin{aligned}
R_{m} & =\frac{-\widetilde{E}_{m}}{2 v_{m}^{2}}\left(V_{m}-v_{m}^{2}-\frac{1}{\sqrt{m}} F_{m}\right)+\frac{3 \widetilde{E}_{m}}{8\left(\xi_{m}\right)^{5 / 2}} \frac{\left(V_{m}-v_{m}^{2}\right)^{2}}{v_{m}^{4}}+\left(\frac{1}{\sqrt{m}}\left(\frac{b_{m}}{v_{m}}-\hat{b}\right)-\frac{b_{m} F_{m}}{2 m v_{m}^{3}}\right) \\
& \equiv R_{m}^{(1)}+R_{m}^{(2)}+R_{m}^{(3)} .
\end{aligned}
$$

It suffices to show

$$
\begin{aligned}
\mathbb{P}\left[U_{m} \leq x\right] & =\Phi(x)+m^{-1 / 2}\left(\frac{A_{1}}{2}-\frac{1}{6}\left(B_{1}-3 A_{1}\right)\left(x^{2}-1\right)\right) \frac{\kappa_{m, 3}}{\left(\kappa_{m, 2}\right)^{3 / 2}} \phi(x)+o\left(m^{-1 / 2}\right), \\
\mathbb{P}\left[t_{m} \leq x\right] & =\mathbb{P}\left[U_{m}+m^{-1 / 2} \hat{b} \leq x\right]+o\left(m^{-1 / 2}\right),
\end{aligned}
$$

uniformly in $x$, as the expansion in (41) easily implies that the expansion for $U_{m}+m^{-1 / 2} \hat{b}$ is the one stated in Proposition 6.1. First, we prove (41). Note that we can not apply Lemma 6.1 directly to $\left(E_{m}, F_{m}\right)$, because it may not possess $\mathbb{I}_{2}$ covariance. For this purpose, we apply a certain transformation and denote

$$
G_{m}=\frac{-u_{m} E_{m}+F_{m}}{\sqrt{w_{m}^{2}-u_{m}^{2}}} .
$$

We want to use Lemma 6.1 with $\left(E_{m}, G_{m}\right)$ and thus need to show that Assumption 6 is satisfied. We easily observe that parts (i) and (ii) are satisfied. Concerning part (iii), we note that the components of $\left(E_{m}, G_{m}\right)$ can be written in the form $\tilde{C}_{m}\left(Z_{m, i}-\mu_{m, i}-B_{m, i}, Z_{m, i}^{2}-\mathbb{E}\left[Z_{m, i}^{2}\right]\right)^{\prime}$ where

$$
\tilde{C}_{m}=\left(\begin{array}{cc}
\frac{1}{v_{m}} & 0 \\
\frac{-u_{m}}{v_{m} \sqrt{w_{m}^{2}-u_{m}^{2}}} & \frac{a_{4}-a_{2}^{2}}{a_{4} \sqrt{w_{m}^{2}-u_{m}^{2}}}
\end{array}\right) .
$$

Also, given $(3+\delta)$ moments of $Z_{m_{i}}^{2}$ and $B_{m, i}$ in Assumption 7 (ii), we get $\left(16 \rho_{m, 3}\right)^{-1} \geq r_{1}>0$ where $\rho_{m, 3}$ belongs to $\left(E_{m}, G_{m}\right)$. Now, let $t_{1}^{2}+t_{2}^{2} \geq r_{1}^{2}$. The structure of above matrix and Assumption 7 (iii),(iv) imply that it suffices to find some $r_{2}>0$ such that

$$
\left(t_{1}+t_{2} \eta_{m}\right)^{2}+\gamma_{m}^{2} t_{2}^{2} \geq r_{2}^{2}
$$


where $\left|\eta_{m}\right| \leq \eta$ uniformly and $\left|\gamma_{m}\right| \geq \gamma>0$. We choose $\Delta$ such that $0<\Delta<1$ and $\Delta \eta<1$. Then $r_{2}=r_{1} \min \left(\sqrt{1-\Delta^{2} \eta^{2}}, \Delta \gamma\right)$. This is easily seen by conditioning on $\left|t_{2}\right| \geq \Delta r_{1}$ and $\left|t_{2}\right|<\Delta r_{1}$. Thus, we obtain

$$
\mathbb{P}\left[E_{m} \leq y, G_{m} \leq z\right]=\int_{-\infty}^{y} \int_{-\infty}^{z}\left(1+m^{-1 / 2} p_{1}(t, s)\right) \phi(s, t) d s d t+o\left(m^{-1 / 2}\right) .
$$

Note that

$$
U_{m}=E_{m}+m^{-1 / 2}\left[E_{m} G_{m}\right] L_{m}\left[E_{m} G_{m}\right]^{\prime}
$$

where

$$
L_{m}=\frac{-1}{2 v_{m}^{2}}\left(\begin{array}{cc}
u_{m} & \frac{1}{2} \sqrt{w_{m}^{2}-u_{m}^{2}} \\
\frac{1}{2} \sqrt{w_{m}^{2}-u_{m}^{2}} & 0
\end{array}\right) \equiv\left(\begin{array}{cc}
c_{m} & b_{m} / 2 \\
b_{m} / 2 & 0
\end{array}\right)
$$

We get

$$
\mathbb{P}\left[U_{m} \leq x\right]=\iint_{\left\{t+m^{-1 / 2}\left(c_{m} t^{2}+b_{m} t s\right) \leq x\right\}}\left(1+\frac{p_{1}(t, s)}{\sqrt{m}}\right) \phi(t, s) d s d t+o\left(\frac{1}{\sqrt{m}}\right) .
$$

To compute above integral, we rely on Lemma 5 in Babu and Singh (1983) (the proof of this result is provided on pp. 228-229 of Babu and Singh (1984)). Although these results mention only the existence of a certain polynomial, a careful inspection of the proof yields an explicit polynomial in our setting. That is

$$
\begin{aligned}
\mathbb{P}\left[U_{m} \leq x\right] & =\int_{-\infty}^{x} \int\left(1+\frac{p_{1}(v, s)}{\sqrt{m}}\right)\left(1-\frac{2 v c_{m}+b_{m} s}{\sqrt{m}}\right)\left(1+\frac{v\left(c_{m} v^{2}+b_{m} v s\right)}{\sqrt{m}}\right) \phi(v, s) d s d v \\
& +o\left(\frac{1}{\sqrt{m}}\right) .
\end{aligned}
$$

Recalling (36), we observe that several terms cancel in above expression which leads to

$$
\begin{aligned}
\mathbb{P}\left[U_{m} \leq x\right] & =\Phi(x)+\frac{\phi(x)}{\sqrt{m}}\left(2 c_{m}-c_{m}\left(2+x^{2}\right)+\frac{\bar{\chi}_{(3,0)}^{m}\left(1-x^{2}\right)}{6}\right)+o\left(\frac{1}{\sqrt{m}}\right) \\
& =\Phi(x)+\frac{\phi(x)}{\sqrt{m}}\left(-c_{m} x^{2}+\frac{\bar{\chi}_{(3,0)}^{m}\left(1-x^{2}\right)}{6}\right)+o\left(\frac{1}{\sqrt{m}}\right) .
\end{aligned}
$$

Note that Assumption 7 implies

$$
\begin{aligned}
\mathbb{E}\left[\left(Z_{m, i}-\mu_{m, i}-B_{m, i}\right)^{3}\right] & =\left(a_{6}-3 a_{2} a_{4}+2 a_{2}^{3}\right) \mu_{m, i}^{3}+O\left(m^{-1}\right), \\
\operatorname{Var}\left(Z_{m, i}-\mu_{m, i}-B_{m, i}\right) & =\left(a_{4}-a_{2}^{2}\right) \mu_{m, i}^{2}+O\left(m^{-1}\right)
\end{aligned}
$$

uniformly in $i$. Hence, we obtain

$$
\begin{aligned}
\bar{\chi}_{(3,0)}^{m} & =\frac{1}{m v_{m}^{3}} \sum_{i=1}^{m} \mathbb{E}\left[\left(Z_{m, i}-\mu_{m, i}-B_{m, i}\right)^{3}\right]=B_{1} \frac{\kappa_{m, 3}}{\left(\kappa_{m, 2}\right)^{3 / 2}}+O\left(m^{-1}\right), \\
c_{m} & =\frac{\operatorname{Cov}\left(E_{m}, F_{m}\right)}{-2 v_{m}^{2}}=-\frac{A_{1}}{2} \frac{\kappa_{m, 3}}{\left(\kappa_{m, 2}\right)^{3 / 2}}+O\left(m^{-1}\right) .
\end{aligned}
$$

By plugging these values we finish the proof of (41). To prove (42), we note that it suffices to show

$$
\mathbb{P}\left[\left|R_{m}\right| \geq m^{-a}\right]=o\left(m^{-1 / 2}\right)
$$


for some $a>1 / 2$ (that will be chosen later). Indeed, using (43) and the fact that the Edgeworth expansion of $U_{m}+m^{-1 / 2} \hat{b}$ holds uniformly in $x$, we obtain

$$
\begin{aligned}
\mathbb{P}\left[t_{m} \leq x\right] & \leq \mathbb{P}\left[U_{m}+m^{-1 / 2} \hat{b} \leq x+m^{-a}\right]+\mathbb{P}\left[\left|R_{m}\right| \geq m^{-a}\right] \\
& =\mathbb{P}\left[U_{m}+m^{-1 / 2} \hat{b} \leq x\right]+o\left(m^{-1 / 2}\right) .
\end{aligned}
$$

Similarly, we show

$$
\mathbb{P}\left[t_{m} \leq x\right] \geq \mathbb{P}\left[U_{m}+m^{-1 / 2} \hat{b} \leq x\right]+o\left(m^{-1 / 2}\right)
$$

and thus obtain (42). To prove (43), we recall the decomposition in (40):

$$
R_{m}=R_{m}^{(1)}+R_{m}^{(2)}+R_{m}^{(3)} .
$$

We observe that $R_{m}^{(2)}$ (and thus $R_{m}$ ) may not have moments. So, we can not show (43) with a plain application of Markov's inequality. Due to (38) and Assumption 7(iv),(v) we obtain

$$
\mathbb{E}\left[\left(R_{m}^{(1)}+R_{m}^{(3)}\right)^{2}\right] \leq \frac{C}{m^{2}}
$$

for some $C>0$. Remembering the constant $\nu$ in Assumption 7(iv), we define $\tilde{\nu}=\frac{a_{4}-a_{2}^{2}}{a_{4}} \frac{\nu}{2}$. Note that

$$
\begin{aligned}
\mathbb{P}\left[V_{m}<\tilde{\nu}\right] & =\mathbb{P}\left[\frac{1}{m} \sum_{i=1}^{m} Z_{m, i}^{2}<\frac{\nu}{2}\right] \\
& \leq \mathbb{P}\left[\frac{1}{m} \sum_{i=1}^{m}\left(Z_{m, i}^{2}-\mathbb{E}\left[Z_{m, i}^{2}\right]\right)<\frac{-\nu}{2}\right] \\
& \leq \mathbb{P}\left[\left|\frac{1}{m} \sum_{i=1}^{m}\left(Z_{m, i}^{2}-\mathbb{E}\left[Z_{m, i}^{2}\right]\right)\right|>\frac{\nu}{2}\right] \leq C / m
\end{aligned}
$$

for some $C>0$ using Markov's inequality. With $r_{m} \equiv\left(\tilde{\nu} / v_{m}^{2}\right)^{5 / 2}<1$ and $\kappa>1$, this last result together with Hölder's inequality and (38) imply

$$
\begin{aligned}
\mathbb{P}\left[\left|R_{m}^{(2)}\right|>\frac{1}{2} m^{-a}\right] & \leq \mathbb{P}\left[\frac{3\left|E_{m}\right|}{4} \frac{\left(V_{m}-v_{m}^{2}\right)^{2}}{v_{m}^{4}}>r_{m} m^{-a}\right]+\mathbb{P}\left[\left(\xi_{m}\right)^{5 / 2}<r_{m}\right] \\
& \leq C m^{\kappa a} \mathbb{E}\left[\left|E_{m}\right|^{\kappa}\left|V_{m}-v_{m}^{2}\right|^{2 \kappa}\right]+\mathbb{P}\left[V_{m}<\tilde{\nu}\right] \\
& \leq C m^{\kappa a} \mathbb{E}\left[\left|E_{m}\right|^{\kappa p}\right]^{1 / p} \mathbb{E}\left[\left|V_{m}-v_{m}^{2}\right|^{2 \kappa q}\right]^{1 / q}+o\left(m^{-1 / 2}\right) \\
& \leq O\left(m^{-\kappa(1-a)}\right)+o\left(m^{-1 / 2}\right)=o\left(m^{-1 / 2}\right)
\end{aligned}
$$

by choosing $p=4, q=4 / 3, \kappa=9 / 8$ and $a \in(1 / 2,10 / 18)$. The last result combined with (44) imply (43) and we are done.

\subsection{Proofs of the main results}

Recalling (20) and (29), for $r>0$, we denote

$$
\begin{aligned}
& \bar{\sigma}_{n}^{r}=\frac{1}{n} \sum_{i=1}^{n}\left(n \int_{\frac{i-1}{n}}^{\frac{i}{n}} \sigma_{t}^{2} d t\right)^{r / 2} \\
& \widetilde{\sigma}_{n}^{r}=\frac{1}{n / k_{n}} \sum_{i=1}^{n / k_{n}}\left(s_{i}^{2} \frac{n}{k_{n} \psi_{2}^{k_{n}}}+\frac{\psi_{1}^{k_{n}} \omega^{2} n}{\psi_{2}^{k_{n}} k_{n}^{2}}\right)^{r / 2}
\end{aligned}
$$


where $s_{i}^{2}$ was defined in (30). Having defined the necessary notations, we state the following preliminary result.

Lemma 6.2. For $r=2,4,6$, we have

$$
\begin{aligned}
& \bar{\sigma}_{n}^{r}-\bar{\sigma}^{r}=o\left(n^{-1 / 2}\right), \\
& \widetilde{\sigma}_{n}^{r}-\widetilde{\sigma}^{r}=o\left(n^{-1 / 4}\right) .
\end{aligned}
$$

Proof. The first result follows from Lemma 2 in Barndorff-Nielsen and Shephard (2003) and is omitted.

We apply similar arguments and prove the second result. In view of the binomial theorem and (14), it suffices to show

$$
\frac{1}{n / k_{n}} \sum_{i=1}^{n / k_{n}}\left(s_{i}^{2} \frac{n}{k_{n} \psi_{2}^{k_{n}}}\right)^{p}-\sum_{i=1}^{n / k_{n}} \int_{\frac{(i-1) k_{n}}{n}}^{\frac{i k_{n}}{n}} \sigma_{t}^{2 p}=o\left(n^{-1 / 4}\right)
$$

We define

$$
m_{i}^{n}=\inf _{\frac{(i-1) k_{n}}{n} \leq t \leq \frac{i k_{n}}{n}} \sigma_{t} \text { and } M_{i}^{n}=\sup _{\frac{(i-1) k_{n}}{n} \leq t \leq \frac{i k_{n}}{n}} \sigma_{t} .
$$

By definitions of the related terms above, we easily observe that

$$
\left(m_{i}^{n}\right)^{2} \leq s_{i}^{2} \frac{n}{k_{n} \psi_{2}^{k_{n}}} \leq\left(M_{i}^{n}\right)^{2} \text { and } \frac{k_{n}}{n}\left(m_{i}^{n}\right)^{2 p} \leq \int_{\frac{(i-1) k_{n}}{n}}^{\frac{i k_{n}}{n}} \sigma_{t}^{2 p} \leq \frac{k_{n}}{n}\left(M_{i}^{n}\right)^{2 p} .
$$

Then, we observe that the abolute value of the expression in (45) may be bounded by

$$
\mathcal{B}_{n} \equiv \frac{1}{n / k_{n}} \sum_{i=1}^{n / k_{n}}\left(M_{i}^{n}\right)^{2 p}-\left(m_{i}^{n}\right)^{2 p}
$$

Since $\sigma$ is pathwise bounded, we easily get

$$
\mathcal{B}_{n} \leq \frac{C}{n / k_{n}} \sum_{i=1}^{n / k_{n}}\left(M_{i}^{n}-m_{i}^{n}\right) .
$$

By definition of the supremum/infimum, there exists $t_{i}^{n}$ and $s_{i}^{n}$ in $\left[(i-1) k_{n} / n, i k_{n} / n\right]$ such that

$$
\left(M_{i}^{n}-m_{i}^{n}\right) \leq\left|\sigma_{t_{i}^{n}}-\sigma_{s_{i}^{n}}\right|+2 / n .
$$

Now, since $k_{n}$ divides $n$, Assumption 2 implies that $\mathcal{B}_{n}=o\left(\sqrt{k_{n} / n}\right)$ which finishes the proof.

We are now ready to prove the main theorems in this paper.

Proof of Theorem 3.1. We start with the proof of part (1) of Theorem 3.1. We recall $Y=X$ with the drift $b=0$ for this theorem and note that we may write the $t$-statistic in (6) for the realized volatility as in (37) by choosing $m=n, B_{n, i}=0$ and

$$
Z_{n, i}=\left|\sqrt{n} \Delta_{i}^{n} X\right|^{2}=\left(\alpha_{m, i} u_{m, i}+\beta_{m, i}\right)^{2}
$$

where $u_{n, i} \sim N(0,1), \beta_{m, i}=0$ and

$$
\mu_{n, i}=\mathbb{E}\left[Z_{n, i}\right]=\alpha_{n, i}^{2}=n \int_{\frac{i-1}{n}}^{\frac{i}{n}} \sigma_{t}^{2} d t
$$


We intend to utilize Proposition 6.1 and observe that Assumption 7(i),(ii), (iv), (v) are obviously satisfied under Assumption 2. Concerning 7(iii), we have

$$
\left(Z_{m, i}-\mu_{m, i}-B_{m, i}, Z_{m, i}^{2}-\mathbb{E}\left[Z_{m, i}^{2}\right]\right)=\left(\alpha_{n, i}^{2}\left(u_{n, i}^{2}-1\right), \alpha_{n, i}^{4}\left(u_{n, i}^{4}-3\right)\right) .
$$

The result follows since $\alpha_{n, i}^{2} \geq \alpha>0$ for all $n$ and $i$ under Assumption 2 .

Having verified the conditions, we look at the expansion in Proposition 6.1 and observe that

$$
\frac{\kappa_{n, 3}}{\left(\kappa_{n, 2}\right)^{3 / 2}}=\frac{\bar{\sigma}_{n}^{6}}{\left(\bar{\sigma}_{n}^{4}\right)^{3 / 2}}
$$

Then, we apply Lemma 6.2 and finish the proof with

$$
\frac{\bar{\sigma}_{n}^{6}}{\left(\bar{\sigma}_{n}^{4}\right)^{3 / 2}}=\sigma_{6,4}+o\left(n^{-1 / 2}\right) .
$$

Similar arguments apply to results in part (2) of Theorem 3.1 i.e., the bootstrap part. Given that in the statement of part (2) of Theorem 3.1) we supposed that the Cramer's condition is satisfied, we only need to verify Assumptions 7(i), (ii), (iv) and (v). It is easy to see that it is the case. In particular, note that we may write the bootstrap $t$-statistic in (10) as in (37) form by choosing $m=n$, $B_{m, i}=0$ and

$$
Z_{m, i}=\left(\alpha_{m, i} u_{m, i}+\beta_{m, i}\right)^{2},
$$

where $\alpha_{m, i}=\sqrt{n} \Delta_{i}^{n} Y, u_{m, i}=v_{i}$ and $\beta_{m, i}=0$ such that the bootstrap external random variable $v_{i} \sim$ i.i.d. with moments given by $a_{s}^{*}=\mathbb{E}^{*}\left|v_{i}\right|^{s}$.

Proof of Proposition 3.1. Let $v_{i}=\sqrt{\eta_{i}} \sim$ i.i.d. with the same distribution as in Proposition 3.1. Since $\eta_{i}$ (and thus $v_{i}$ ) has a density, Cramer's condition for $v$ is satisfied. The discussion before Proposition 4.5 in GM(2009) means that the following moment conditions are sufficient for a secondorder asymptotic refinement:

$$
\mathbb{E}\left[v^{2}\right]=1, \quad \mathbb{E}\left[v^{4}\right]=\frac{31}{25}, \quad \text { and } \mathbb{E}\left[v^{6}\right]=\frac{31}{25} \frac{37}{25} .
$$

We note that $\mathbb{E}\left[v^{2 r}\right]=\mathbb{E}\left[\eta^{r}\right]$ for $r=1,2,3$. For the gamma distribution with parameters $\alpha>0$ and $\beta>0$, it is well-known that

$$
\mathbb{E}[\eta]=\frac{\alpha}{\beta}, \quad \mathbb{E}\left[\eta^{2}\right]=\frac{\alpha(\alpha+1)}{\beta^{2}} \quad \text { and } \mathbb{E}\left[\eta^{3}\right]=\frac{\alpha(\alpha+1)(\alpha+2)}{\beta^{3}} .
$$

Solving these equations in $\alpha$ and $\beta$ leads to $\alpha=\beta=\frac{25}{6}$.

Proof of Remark 3. We proceed as in the proof of part (1) of Theorem 3.1. In particular, we recall $Y=X$ for this case and note that we may write the $t$-statistic in (6) for the realized volatility as in (37) by choosing $m=n, B_{n, i}=0$ and

$$
Z_{n, i}=\left|\sqrt{n} \Delta_{i}^{n} X\right|^{2} .
$$

Since $\sqrt{n} \Delta_{i}^{n} X$ is normally distributed, we obtain

$$
\mu_{n, i} \equiv \mathbb{E}\left[Z_{n, i}\right]=n \int_{\frac{i-1}{n}}^{\frac{i}{n}} \sigma_{t}^{2} d t+\frac{b^{2}}{n} .
$$

We intend to utilize Proposition 6.1 and observe that Assumption 7(i),(ii), (iv) are obviously satisfied. Concerning $7(\mathrm{v})$, we observe that $b_{n}=b^{2}$ and $v_{n}^{2}=\bar{\sigma}_{n}^{4}+O\left(n^{-1 / 2}\right)$. Hence, Lemma 6.2 yields $\hat{b}=b^{2} / \bar{\sigma}^{4}$. Since we assume the existence of the expansions, we will not verify the Cramer's condition stated in Assumption 7(iii). 
Next, we move to the proof of the result for the pre-averaging estimator.

Proof of Theorem 3.2. For the proof of part (1) of this theorem, our first aim is to write the main part of the pre-averaging estimator in the form given by (37). For this purpose, we denote $m=n / k_{n}$ and recalling (30), we write

$$
\begin{aligned}
Z_{m, i} & =\frac{n}{k_{n} \psi_{2}^{k_{n}}}\left(\bar{Y}_{(i-1) k_{n}}\right)^{2}, \mu_{m, i}=\frac{n}{k_{n} \psi_{2}^{k_{n}}}\left(s_{i}^{2}+\frac{\psi_{1}^{k_{n}} \omega^{2}}{k_{n}}\right), \\
B_{m, i} & =\frac{n}{k_{n}} \frac{\psi_{1}^{k_{n}}}{2 k_{n}^{2} \psi_{2}^{k_{n}}} \sum_{j=(i-1) k_{n}+1}^{i k_{n}-1}\left(\left|\Delta_{j}^{n} \epsilon\right|^{2}-2 \omega^{2}\right) .
\end{aligned}
$$

Then,

$$
T_{n}=t_{m}+R_{n}
$$

where $T_{n}$ and $t_{m}$ were defined in (17) and (37), respectively, and the reminder term $R_{n}$ is given by

$$
R_{n}=\tilde{R}_{n} / \sqrt{\hat{V}_{n}}
$$

with

$$
\begin{aligned}
\tilde{R}_{n} & =n^{1 / 4}\left(\frac{1}{\psi_{2}^{k_{n}}} \sum_{i=1}^{d_{n}} s_{i}^{2}-\int_{0}^{1} \sigma_{t}^{2} d t\right)-\frac{n^{1 / 4} \psi_{1}^{k_{n}}}{2 k_{n}^{2} \psi_{2}^{k_{n}}}\left(\sum_{i=1}^{n}\left(\left|\Delta_{i}^{n} Y\right|^{2}-\left|\Delta_{i}^{n} \epsilon\right|^{2}\right)+\sum_{j=1}^{d_{n}}\left(\left|\Delta_{j k_{n}}^{n} \epsilon\right|^{2}-2 \omega^{2}\right)\right) \\
& \equiv \tilde{R}_{n}^{(1)}+\tilde{R}_{n}^{(2)} .
\end{aligned}
$$

First, assume that we can apply Proposition 6.1 for $t_{m}$ (we will later show that $R_{n}$ is negligible). Proceeding as in the proof of Theorem 3.1, we get

$$
\frac{\kappa_{n, 3}}{\left(\kappa_{n, 2}\right)^{3 / 2}}=\frac{\tilde{\sigma}_{n}^{6}}{\left(\tilde{\sigma}_{n}^{4}\right)^{3 / 2}} .
$$

Then, we will be done due to Lemma 6.2. Now, we verify that the assumptions of Proposition 6.1 are satisfied. Clearly, Assumptions 7(i), (iii), (iv) and (v) hold true. Next, we check Assumption 7(ii). Since $\bar{X}_{(i-1) k_{n}}$ and $\bar{\epsilon}_{(i-1) k_{n}}$ are independent and have 0 means, we get

$$
\mathbb{E}\left[\left(\bar{Y}_{(i-1) k_{n}}\right)^{4}\right]=\mathbb{E}\left[\left(\bar{X}_{(i-1) k_{n}}\right)^{4}\right]+6 \mathbb{E}\left[\left(\bar{X}_{(i-1) k_{n}}\right)^{2}\right] \mathbb{E}\left[\left(\bar{\epsilon}_{(i-1) k_{n}}\right)^{2}\right]+\mathbb{E}\left[\left(\bar{\epsilon}_{(i-1) k_{n}}\right)^{4}\right]
$$

Since the term $\bar{X}_{(i-1) k_{n}}$ is normally distributed with mean 0 and variance $s_{i}^{2}$, its moments are wellknown. However, the term $\bar{\epsilon}_{(i-1) k_{n}}$ may not be normally distributed and needs a careful treatment. To deal with the moments of the noise term, we define $h\left(j / k_{n}\right)=g\left((j+1) / k_{n}\right)-g\left(j / k_{n}\right)$ for $1 \leq j \leq k_{n}-1$. It is easy to see that

$$
\bar{\epsilon}_{i}=\sum_{j=0}^{k_{n}-1}-h\left(j / k_{n}\right) \epsilon_{\frac{i+j}{n}} .
$$

For $p=4,6$, let's denote

$$
\psi_{p}^{k_{n}}=k_{n}^{p-1} \sum_{j=0}^{k_{n}-1} h\left(j / k_{n}\right)^{p} .
$$


We note that since $g$ is Lipschitz continuous, we have $\psi_{p}^{n}=O(1)$ for $p=4,6$. Let's also denote $p$-th absolute moment of $\epsilon_{\frac{i}{n}}$ with $m_{p}$. A simple calculation shows that

$$
\mathbb{E}\left[\left(\bar{\epsilon}_{i}\right)^{4}\right]=\frac{3\left(\psi_{1}^{k_{n}}\right)^{2} \omega^{4}}{k_{n}^{2}}+\frac{\left(m_{4}-3 \omega^{4}\right) \psi_{4}^{k_{n}}}{k_{n}^{3}} .
$$

At this stage, we easily get

$$
\mathbb{E}\left[Z_{m, i}^{2}\right]=\frac{n^{2}}{\left(k_{n}\right)^{2}\left(\psi_{2}^{k_{n}}\right)^{2}} \mathbb{E}\left[\left(\bar{Y}_{(i-1) k_{n}}^{n}\right)^{4}\right]=\frac{3 n^{2}}{\left(k_{n}\right)^{2}\left(\psi_{2}^{k_{n}}\right)^{2}}\left(s_{i}^{2}+\frac{\psi_{1}^{k_{n}} \omega^{2}}{k_{n}}\right)^{2}+\frac{3 n^{2}\left(m_{4}-3\right) \psi_{4}^{k_{n}}}{k_{n}^{5}\left(\psi_{2}^{k_{n}}\right)^{2}}
$$

and, due to $m=n / k_{n}$, this leads to

$$
\left|\mathbb{E}\left[Z_{m, i}^{2}\right]-\frac{a_{4}}{a_{2}^{2}} \mu_{m, i}^{2}\right| \leq \frac{C}{m}
$$

Similarly, it is possible to show the assumption related to

$$
\mathbb{E}\left[Z_{m, i}^{3}\right]=\frac{n^{3}}{\left(k_{n}\right)^{3}\left(\psi_{2}^{k_{n}}\right)^{3}} \mathbb{E}\left[\left(\bar{Y}_{(i-1) k_{n}}\right)^{6}\right]
$$

In this case, crucial steps are to use (47) and

$$
\mathbb{E}\left[\left(\bar{\epsilon}_{i}\right)^{6}\right]=\frac{15\left(\psi_{1}^{k_{n}}\right)^{3} \omega^{4}}{k_{n}^{3}}+\frac{\left(m_{6}-15 \omega^{4}\right) \psi_{6}^{k_{n}}}{k_{n}^{5}}+\frac{O(1)}{k_{n}^{5}} .
$$

Concerning the condition for $\mathbb{E}\left[Z_{m, i} B_{m, i}\right]$, we observe that

$$
\mathbb{E}\left[Z_{m, i} B_{m, i}\right]=\frac{n^{2} \psi_{1}^{k_{n}}}{2 k_{n}^{4}\left(\psi_{2}^{k_{n}}\right)^{2}} \sum_{j=(i-1) k_{n}+1}^{i k_{n}-1} \mathbb{E}\left[\left(\bar{\epsilon}_{(i-1) k_{n}}\right)^{2}\left(\left(\Delta_{j}^{n} \epsilon\right)^{2}-2 \omega^{2}\right)\right] .
$$

To compute this expression, for each $j$ in above range, we find that

$$
\begin{aligned}
\mathbb{E}\left[\left(\bar{\epsilon}_{(i-1) k_{n}}\right)^{2}\left(\left(\Delta_{j}^{n} \epsilon\right)^{2}-2 \omega^{2}\right)\right]= & \left(h\left(j / k_{n}\right)^{2}+h\left((j-1) / k_{n}\right)^{2}\right)\left(m_{4}-\omega^{4}\right) \\
& +4 h\left(j / k_{n}\right) h\left((j-1) / k_{n}\right) \omega^{4}=O\left(1 / k_{n}^{2}\right)
\end{aligned}
$$

where we exploited the Lipschitz continuity of $g$. This easily leads to $\mathbb{E}\left[Z_{m, i} B_{m, i}\right]=O\left(1 / k_{n}\right)=O(1 / m)$. Lastly, we get

$$
\mathbb{E}\left[Z_{m, i}^{2} B_{m, i}\right]=\frac{n^{3} \psi_{1}^{k_{n}}}{2 k_{n}^{5}\left(\psi_{2}^{k_{n}}\right)^{3}} \sum_{j=(i-1) k_{n}+1}^{i k_{n}-1} \mathbb{E}\left[\left\{6\left(\bar{X}_{(i-1) k_{n}}\right)^{2}\left(\bar{\epsilon}_{(i-1) k_{n}}\right)^{2}+\left(\bar{\epsilon}_{(i-1) k_{n}}\right)^{4}\right\}\left(\left(\Delta_{j}^{n} \epsilon\right)^{2}-2 \omega^{2}\right)\right]
$$

In this case, the condition is verified by noting that the additional term satisfies

$$
\mathbb{E}\left[\left(\bar{\epsilon}_{(i-1) k_{n}}\right)^{4}\left(\left(\Delta_{j}^{n} \epsilon\right)^{2}-2 \omega^{2}\right)\right]=O\left(1 / k_{n}^{3}\right) .
$$

Next, we show that the remainder term defined in (46) does not influence the Edgeworth expansion of $T_{n}$. We deal with this term similar to (40). Assumption 4 implies $\tilde{R}_{n}^{(1)}=O\left(n^{-1 / 4-\delta}\right)$ for some $\delta>0$. Moreover, we have

$$
\mathbb{E}\left[\left(\tilde{R}_{n}^{(2)}\right)^{2}\right] \leq C / n
$$


for some $C>0$. Combining these results leads to

$$
\mathbb{P}\left[\left|R_{n}\right|>n^{-1 / 4-\delta / 2}\right]=o\left(n^{-1 / 4}\right) .
$$

This implies that $R_{n}$ has no effect on the Edgeworth expansion of $T_{n}$.

For the proof of results in part 2) of Theorem 3.2, similar arguments as in the bootstrap part of Theorem 3.1 (no noise case) apply. In particular, one can see that, here we may write the bootstrap $t$-statistic in (19) as in (37) form by choosing $m=n / k_{n}, B_{m, i}=0$ and

$$
Z_{m, i}=\left(\alpha_{m, i} u_{m, i}+\beta_{m, i}\right)^{2},
$$

where $\alpha_{m, i}=\left(\frac{n}{k_{n} \psi_{2}^{k_{n}}}\right)^{1 / 2} \bar{Y}_{i k_{n}}, u_{m, i}=v_{i}$ and $\beta_{m, i}=0$ such that the bootstrap external random variable $v_{i} \sim$ i.i.d. with moments given by $a_{s}^{*}=\mathbb{E}^{*}\left|v_{i}\right|^{s}$. In particular, $\mu_{m, i}=a_{2} \frac{n}{k_{n} \psi_{2}^{k_{n}}} \bar{Y}_{i k_{n}}^{2}$. This complete the proof.

Proof of Corollary 3.1. Immediate given Proposition 6.1 and the proof of part a) of Theorem 3.2 .

Proof of Proposition 3.2. Result follows given part a) of Theorem 3.2 in conjunction with Corollary 3.1 by applying Proposition 3.1 .

\section{References}

Aït-Sahalia, Y., P. A. Mykland, and L. Zhang (2011). Ultra high frequency volatility estimation with dependent microstructure noise. Journal of Econometrics 160(1), 160-175.

Andersen, T. G., T. Bollerslev, and F. X. Diebold (2010). Parametric and nonparametric volatility measurement. In L. P. Hansen and Y. Ait-Sahalia (Eds.), Handbook of Financial Econometrics, pp. 67-138. Amsterdam: North-Holland.

Andersen, T. G., T. Bollerslev, F. X. Diebold, and P. Labys (2001). The distribution of realized exchange rate volatility. Journal of the American Statistical Association 96(453), 42-55.

Babu, G. J. and K. Singh (1983). Inference on means using the bootstrap. Annals of Statistics 11(3), 999-1003.

Babu, G. J. and K. Singh (1984). On one term Edgeworth correction by Efron's bootstrap. Sankhyā Series A 46(2), 219-232.

Bandi, F. M. and J. R. Russell (2008). Microstructure noise, realized variance, and optimal sampling. Review of Economic Studies 75(2), 339-369.

Barndorff-Nielsen, O. E., S. E. Graversen, J. Jacod, and N. Shephard (2006). Limit theorems for bipower variation in financial econometrics. Econometric Theory 22, 677-719.

Barndorff-Nielsen, O. E. and N. Shephard (2002). Econometric analysis of realized volatility and its use in estimating stochastic volatility models. Journal of the Royal Statistical Society: Series B $64(2), 253-280$.

Barndorff-Nielsen, O. E. and N. Shephard (2003). Realized power variation and stochastic volatility models. Bernoulli 9, 243-265. 
Barndorff-Nielsen, O. E. and N. Shephard (2007). Variation, jumps, market frictions and high frequency data in financial econometrics. In R. Blundell, P. Torsten, and W. K. Newey (Eds.), Advances in Economics and Econometrics: Theory and Applications, Ninth World Congress, Volume III, pp. 328-372. Cambridge: Cambridge University Press.

Bhattacharya, R. N. and R. R. Rao (1986). Normal approximation and asymptotic expansions. Robert E. Krieger Publishing Co., Inc., Melbourne, FL.

Christensen, K., S. Kinnebrock, and M. Podolskij (2010). Pre-averaging estimators of the ex-post covariance matrix in noisy diffusion models with non-synchronous data. Journal of Econometrics $159(1), 116-133$.

Comte, F. and E. Renault (1998). Long memory in continuous-time stochastic volatility models. Mathematical Finance 8(4), 291-323.

Davidson, R. and E. Flachaire (2001). The wild bootstrap, tamed at last. Working Paper Darp58, STICERD, London School of Economics.

Gonçalves, S., U. Hounyo, and N. Meddahi (2014). Bootstrap inference for pre-averaged realized volatility based on non-overlapping returns. Journal of Financial Econometrics 12(4), 679-707.

Gonçalves, S. and N. Meddahi (2008). Edgeworth corrections for realized volatility. Econometric Reviews 27(1-3), 139-162.

Gonçalves, S. and N. Meddahi (2009). Bootstrapping realized volatility. Econometrica r7(1), 283-306.

Hall, P. (1992). The bootstrap and Edgeworth expansion. Springer Series in Statistics. Springer-Verlag, New York.

Hansen, P. R. and A. Lunde (2006). Realized variance and market microstructure noise. Journal of Business and Economic Statistics 24(2), 127-161.

Hautsch, N. and M. Podolskij (2013). Pre-averaging based estimation of quadratic variation in the presence of noise and jumps: Theory, implementation, and empirical evidence. Journal of Business and Economic Statistics 31(2), 165-183.

Hounyo, U. (2013). Bootstrapping realized volatility and realized beta under a local Gaussianity assumption. Research paper 2013-30, CREATES, Aarhus University.

Hounyo, U., S. Gonçalves, and N. Meddahi (2013). Bootstrapping pre-averaged realized volatility under market microstructure noise. Research paper 2013-28, CREATES, Aarhus University.

Huang, X. and G. Tauchen (2005). The relative contribution of jumps to total price variance. Journal of Financial Econometrics 3(4), 456-499.

Jacod, J., Y. Li, P. A. Mykland, M. Podolskij, and M. Vetter (2009). Microstructure noise in the continuous case: the pre-averaging approach. Stochastic Processes and their Applications 119(7), 2249-2276.

Jacod, J. and P. E. Protter (1998). Asymptotic error distributions for the Euler method for stochastic differential equations. Annals of Probability 26(1), 267-307.

Lahiri, S. N. (2003). Resampling methods for dependent data. Springer Series in Statistics. SpringerVerlag, New York. 
Liu, R. Y. (1988). Bootstrap procedures under some non-i.i.d. models. Annals of Statistics 16(4), $1696-1708$.

Mammen, E. (1993). Bootstrap and wild bootstrap for high-dimensional linear models. Annals of Statistics. 21(1), 255-285.

Meddahi, N. (2002). A theoretical comparison between integrated and realized volatility. Journal of Applied Econometrics 17(5), 479-508.

Podolskij, M. and M. Vetter (2009). Estimation of volatility functionals in the simultaneous presence of microstructure noise and jumps. Bernoulli 15(3), 634-658.

Podolskij, M. and N. Yoshida (2014). Edgeworth expansion for functionals of continuous diffusion processes. Preprint.

Yoshida, N. (2013). Martingale expansion in mixed normal limit. Stochastic Processes and their Applications 123(3), 887-933.

Zhang, L. (2006). Efficient estimation of stochastic volatility using noisy observations: A multi-scale approach. Bernoulli 12(6), 1019-1043.

Zhang, L., P. A. Mykland, and Y. Aït-Sahalia (2005). A tale of two time scales: determining integrated volatility with noisy high-frequency data. Journal of the American Statistical Association 100(472), 1394-1411.

Zhang, L., P. A. Mykland, and Y. Aït-Sahalia (2011). Edgeworth expansions for realized volatility and related estimators. Journal of Econometrics 160(1), 190-203. 
2015-04: $\quad$ Maria Eugenia Sanin, Maria Mansanet-Bataller and Francesco Violante: Understanding volatility dynamics in the EU-ETS market

2015-05: Peter Christoffersen and Xuhui (Nick) Pan: Equity Portfolio Management Using Option Price Information

2015-06: Peter Christoffersen and Xuhui (Nick) Pan: Oil Volatility Risk and Expected Stock Returns

2015-07: Peter Christoffersen, Bruno Feunou and Yoontae Jeon: Option Valuation with Observable Volatility and Jump Dynamics

2015-08: $\quad$ Alfonso Irarrazabal and Juan Carlos Parra-Alvarez: Time-varying disaster risk models: An empirical assessment of the Rietz-Barro hypothesis

2015-09: Daniela Osterrieder, Daniel Ventosa-Santaulària and Eduardo Vera-Valdés: Unbalanced Regressions and the Predictive Equation

2015-10: Laurent Callot, Mehmet Caner, Anders Bredahl Kock and Juan Andres Riquelme: Sharp Threshold Detection Based on Sup-norm Error rates in Highdimensional Models

2015-11: $\quad$ Arianna Agosto, Giuseppe Cavaliere, Dennis Kristensen and Anders Rahbek: Modeling corporate defaults: Poisson autoregressions with exogenous covariates (PARX)

2015-12: Tommaso Proietti, Martyna Marczak and Gianluigi Mazzi: EuroMInd-D: A Density Estimate of Monthly Gross Domestic Product for the Euro Area

2015-13: $\quad$ Michel van der Wel, Sait R. Ozturk and Dick van Dijk: Dynamic Factor Models for the Volatility Surface

2015-14: $\quad$ Tim Bollerslev, Andrew J. Patton and Rogier Quaedvlieg: Exploiting the Errors: A Simple Approach for Improved Volatility Forecasting

2015-15: $\quad$ Hossein Asgharian, Charlotte Christiansen and Ai Jun Hou: Effects of Macroeconomic Uncertainty upon the Stock and Bond Markets

2015-16: $\quad$ Markku Lanne, Mika Meitz and Pentti Saikkonen: Identification and estimation of non-Gaussian structural vector autoregressions

2015-17: Nicholas M. Kiefer and C. Erik Larson: Counting Processes for Retail Default Modeling

2015-18: Peter Reinhard Hansen: A Martingale Decomposition of Discrete Markov Chains

2015-19: $\quad$ Peter Reinhard Hansen, Guillaume Horel, Asger Lunde and Ilya Archakov: A Markov Chain Estimator of Multivariate Volatility from High Frequency Data

2015-20: Henri Nyberg and Harri Pönkä: International Sign Predictability of Stock Returns: The Role of the United States

2015-21: Ulrich Hounyo and Bezirgen Veliyev: Validity of Edgeworth expansions for realized volatility estimators 